\title{
Marchenko imaging
}

\author{
Kees Wapenaar ${ }^{1}$, Jan Thorbecke ${ }^{1}$, Joost van der Neut ${ }^{1}$, Filippo Broggini ${ }^{2}$, Evert Slob ${ }^{1}$, \\ and Roel Snieder ${ }^{3}$
}

\begin{abstract}
Traditionally, the Marchenko equation forms a basis for 1D inverse scattering problems. A 3D extension of the Marchenko equation enables the retrieval of the Green's response to a virtual source in the subsurface from reflection measurements at the earth's surface. This constitutes an important step beyond seismic interferometry. Whereas seismic interferometry requires a receiver at the position of the virtual source, for the Marchenko scheme it suffices to have sources and receivers at the surface only. The underlying assumptions are that the medium is lossless and that an estimate of the direct arrivals of the Green's function is available. The Green's function retrieved with the 3D Marchenko scheme contains accurate internal multiples of the
\end{abstract}

inhomogeneous subsurface. Using source-receiver reciprocity, the retrieved Green's function can be interpreted as the response to sources at the surface, observed by a virtual receiver in the subsurface. By decomposing the 3D Marchenko equation, the response at the virtual receiver can be decomposed into a downgoing field and an upgoing field. By deconvolving the retrieved upgoing field with the downgoing field, a reflection response is obtained, with virtual sources and virtual receivers in the subsurface. This redatumed reflection response is free of spurious events related to internal multiples in the overburden. The redatumed reflection response forms the basis for obtaining an image of a target zone. An important feature is that spurious reflections in the target zone are suppressed, without the need to resolve first the reflection properties of the overburden.

\section{INTRODUCTION}

The Marchenko equation has since long been used by mathematical physicists as a basis for 1D inverse scattering theory (Marchenko, 1955; Burridge, 1980; Lamb, 1980; Ge, 1987; Chadan and Sabatier, 1989). It relates the reflection response, measured on one side of a lossless 1D medium, to a field inside that medium, which, in turn, is related to the scattering potential in the medium. Inverse scattering methods derived from the Marchenko equation fully account for internal multiple scattering. Broggini and Snieder (2012) discuss an interesting link between the Marchenko equation and seismic interferometry. They show that, by using the Marchenko equation, the Green's function between an arbitrary virtualsource position inside the $1 \mathrm{D}$ medium and a receiver at the surface can be retrieved from the reflection response measured at the surface of that medium. This constitutes an important step beyond seis- mic interferometry. To retrieve a Green's function with seismic interferometry, the 1D medium should be illuminated from both sides and a physical receiver should be present inside the medium, at the position of the virtual source. For example, the 1D version of the virtual-source method proposed by Bakulin and Calvert (2006) requires a receiver in a borehole, illuminated from above and below (Curtis et al., 2006); the fact that in practice the illumination occurs only from above implies that the retrieved Green's function contains spurious multiples (Snieder et al., 2006). In contrast to this, the scheme proposed by Broggini and Snieder (2012) requires no physical receivers inside the medium, and illumination from one side suffices. Nevertheless, the retrieved Green's function contains accurate internal multiple reflections. A restriction is that the virtual-source position is not defined in depth but in one-way traveltime. Hence, whereas the Green's function is retrieved from the reflection response alone (i.e., without any knowledge about the

Manuscript received by the Editor 9 August 2013; revised manuscript received 7 January 2014; published online 22 May 2014.

${ }^{1}$ Delft University of Technology, Department of Geoscience and Engineering, Delft, The Netherlands. E-mail: c.p.a.wapenaar@tudelft.nl; j.w.thorbecke@ tudelft.nl; j.r.vanderneut@tudelft.nl; e.c.slob@tudelft.nl.

${ }^{2}$ Formerly Colorado School of Mines, Center for Wave Phenomena, Golden, Colorado, USA; presently ETH Zürich, Institute of Geophysics, Exploration and Environmental Geophysics, Zürich, Switzerland. E-mail: filippo.broggini@erdw.ethz.ch.

${ }^{3}$ Colorado School of Mines, Center for Wave Phenomena, Golden, Colorado, USA. E-mail: rsnieder@mines.edu.

(C) 2014 Society of Exploration Geophysicists. All rights reserved. 
medium), determining the position of the virtual source requires velocity information to perform time-depth conversion (a smooth velocity model usually suffices for this purpose).

We have recently extended the 1D approach of Broggini and Snieder (2012) to three dimensions (Wapenaar et al., 2012, 2014). To this end, we derived a 3D Marchenko equation, which relates the reflection response at the surface of a lossless 3D inhomogeneous medium to a field inside that medium. This field is called the focusing function. It is obtained from the reflection response by solving the 3D Marchenko equation via an iterative procedure. Subsequently, the Green's function between a virtual source inside the medium and receivers at the surface is retrieved from the reflection response and the focusing function. Assuming the reflection response at the surface is well sampled, this recovered Green's function properly contains the internal multiples of the 3D inhomogeneous medium. As in the $1 \mathrm{D}$ case, no physical receiver is required at the position of the virtual source. Apart from the reflection response at the surface, the method requires an estimate of the direct arrivals (including triplications in case of multipathing) between the virtual-source position and the receivers at the surface. This requirement replaces the need for time-depth conversion in the 1D scheme.

In this paper, we briefly review the 3D Marchenko equation and the method for retrieving the Green's function from the reflection response at the surface and an estimate of the direct arrivals. We interpret the retrieved Green's function as the response to sources at the surface, observed by a virtual receiver in the subsurface (which, via reciprocity, is the same as the response to a virtual source in the subsurface, observed by receivers at the surface). Next, we discuss decomposition of the Marchenko equation and show how this can be used to retrieve the downgoing and upgoing parts of the Green's function at a virtual receiver in the subsurface, in response to sources at the surface. These retrieved downgoing and upgoing Green's functions in the subsurface form the basis for redatuming and imaging. Unlike standard redatuming and imaging, which uses approximations of the downgoing and upgoing wavefields in the subsurface, our new method uses true downgoing and upgoing wavefields (i.e., Green's functions, including accurate internal multiple reflections). This implies that the internal multiples contribute to imaging the reflectivity and that the obtained image will be free of spurious events related to the internal multiples.

Homogeneous half-space

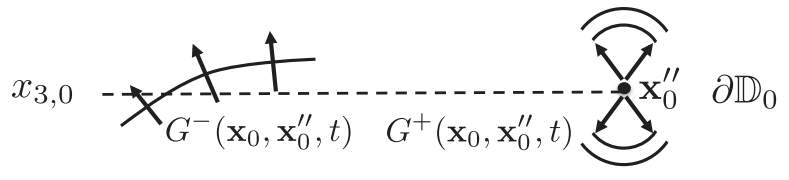

Actual inhomogeneous medium

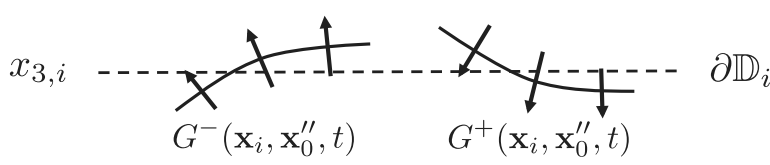

Actual inhomogeneous half-space

Figure 1. Downgoing and upgoing components of the Green's function $G\left(\mathbf{x}, \mathbf{x}_{0}^{\prime \prime}, t\right)$ of the 3D wave equation in the actual inhomogeneous medium.
We illustrate this with some simple examples. In a companion paper (Broggini et al., 2014), we discuss various aspects of this new imaging approach in more detail, including its sensitivity to errors in the estimated direct arrivals.

\section{GREEN'S FUNCTIONS AND FOCUSING FUNCTIONS}

We start by reviewing Green's functions, focusing functions, and their mutual relations in a 3D inhomogeneous medium (the details of the derivation are given in Appendix A). Consider the configuration depicted in Figure 1. It consists of a 3D inhomogeneous lossless half-space below a transparent boundary $\partial \mathbb{D}_{0}$ and a homogeneous half-space above that boundary. Spatial coordinates are denoted as $\mathbf{x}=\left(x_{1}, x_{2}, x_{3}\right)$, with $x_{3}$ positive in the lower half-space and negative in the upper half-space. Boundary $\partial \mathbb{D}_{0}$ is defined as $x_{3}=x_{3,0}=0$. For convenience, coordinates at $\partial \mathbb{D}_{0}$ are denoted as $\mathbf{x}_{0}=\left(\mathbf{x}_{\mathrm{H}}, x_{3,0}\right)$, with $\mathbf{x}_{\mathrm{H}}=\left(x_{1}, x_{2}\right)$. Similarly, coordinates at an arbitrary depth level $\partial \mathbb{D}_{i}$ are denoted as $\mathbf{x}_{i}=\left(\mathbf{x}_{\mathrm{H}}, x_{3, i}\right)$, where $x_{3, i}$ denotes the depth of $\partial \mathbb{D}_{i}$.

\section{Green's functions}

We define the Green's function $G\left(\mathbf{x}, \mathbf{x}_{0}^{\prime \prime}, t\right)$ as the causal solution of the scalar wave equation in the actual inhomogeneous medium, with a source at $\mathbf{x}_{0}^{\prime \prime}$, according to

$$
\rho \nabla \cdot\left(\frac{1}{\rho} \nabla G\right)-\frac{1}{c^{2}} \frac{\partial^{2} G}{\partial t^{2}}=-\rho \delta\left(\mathbf{x}-\mathbf{x}_{0}^{\prime \prime}\right) \frac{\partial \delta(t)}{\partial t} .
$$

Here, $c=c(\mathbf{x})$ and $\rho=\rho(\mathbf{x})$ are the propagation velocity and mass density of the inhomogeneous medium and $t$ denotes time. Defined in this way, the Green's function $G\left(\mathbf{x}, \mathbf{x}_{0}^{\prime \prime}, t\right)$ is the response (in terms of acoustic pressure) to an impulsive point source of volume injection rate at $\mathbf{x}_{0}^{\prime \prime}$, observed at $\mathbf{x}$ (de Hoop, 1995). In the following, $\mathbf{x}_{0}^{\prime \prime}$ is chosen just above $\partial \mathbb{D}_{0}$, see Figure 1 . Hence, $\mathbf{x}_{0}^{\prime \prime}=$ $\left(\mathbf{x}_{\mathrm{H}}^{\prime \prime}, x_{3,0}-\epsilon\right)$, with $\epsilon \rightarrow 0$. The Green's function is decomposed into downgoing and upgoing fields, which are mutually coupled by the inhomogeneities of the medium below $\partial \mathbb{D}_{0}$. The downgoing and upgoing components at observation point $\mathbf{x}$ are represented by $G^{+}\left(\mathbf{x}, \mathbf{x}_{0}^{\prime \prime}, t\right)$ and $G^{-}\left(\mathbf{x}, \mathbf{x}_{0}^{\prime \prime}, t\right)$, respectively. Assuming the one-way wavefields are pressure-normalized, the two-way Green's function is defined as the superposition of the downgoing and upgoing fields, according to

$$
G\left(\mathbf{x}, \mathbf{x}_{0}^{\prime \prime}, t\right)=G^{+}\left(\mathbf{x}, \mathbf{x}_{0}^{\prime \prime}, t\right)+G^{-}\left(\mathbf{x}, \mathbf{x}_{0}^{\prime \prime}, t\right),
$$

for any $\mathbf{x}$ at or below $\partial \mathbb{D}_{0}$.

The vertical derivative of the downgoing Green's function at $\partial \mathbb{D}_{0}$ (just below the source point) is given by

$$
\left.\partial_{3} G^{+}\left(\mathbf{x}, \mathbf{x}_{0}^{\prime \prime}, t\right)\right|_{x_{3}=x_{3,0}}=-\frac{1}{2} \rho\left(\mathbf{x}_{0}^{\prime \prime}\right) \delta\left(\mathbf{x}_{\mathrm{H}}-\mathbf{x}_{\mathrm{H}}^{\prime \prime}\right) \frac{\partial \delta(t)}{\partial t}
$$

where $\partial_{3}$ stands for $\partial / \partial x_{3}$. The vertical derivative of the upgoing Green's function at $\partial \mathbb{D}_{0}$ is related to the pressure-normalized reflection response of the inhomogeneous medium below $\partial \mathbb{D}_{0}$, according to 


$$
\left.\partial_{3} G^{-}\left(\mathbf{x}, \mathbf{x}_{0}^{\prime \prime}, t\right)\right|_{x_{3}=x_{3,0}}=\frac{1}{2} \rho\left(\mathbf{x}_{0}\right) \frac{\partial R^{\cup}\left(\mathbf{x}_{0}^{\prime \prime}, \mathbf{x}_{0}, t\right)}{\partial t}
$$

(Appendix A, equation A-6). Here, the superscript $\cup$ denotes that $R^{\cup}\left(\mathbf{x}_{0}^{\prime \prime}, \mathbf{x}_{0}, t\right)$ is the reflection response to downgoing waves of the medium below depth level $x_{3,0}$. This is to be distinguished from $R^{\cap}\left(\mathbf{x}_{j}^{\prime}, \mathbf{x}_{j}, t\right)$, introduced in the section "imaging from below," which is the reflection response to upgoing waves of the medium above depth level $x_{3, j}$.

\section{Focusing functions}

Next, we define so-called focusing functions in a reference configuration; see Figure 2 (Slob et al., 2014; Wapenaar et al., 2014). The reference medium is identical to the actual medium above depth level $\partial \mathbb{D}_{i}$ and reflection-free below this depth level. First, consider the focusing function $f_{1}\left(\mathbf{x}, \mathbf{x}_{i}^{\prime}, t\right)$ in Figure 2a. Here, $\mathbf{x}_{i}^{\prime}=\left(\mathbf{x}_{\mathrm{H}}^{\prime}, x_{3, i}\right)$ denotes a focal point at lateral position $\mathbf{x}_{\mathrm{H}}^{\prime}$ anywhere on $\partial \mathbb{D}_{i}$, whereas $\mathbf{x}$ represents an observation point anywhere in the medium. Analogous to equation 2, the focusing function is written as the superposition of (mutually coupled) pressure-normalized downgoing and upgoing components at observation point $\mathbf{x}$, according to

$$
f_{1}\left(\mathbf{x}, \mathbf{x}_{i}^{\prime}, t\right)=f_{1}^{+}\left(\mathbf{x}, \mathbf{x}_{i}^{\prime}, t\right)+f_{1}^{-}\left(\mathbf{x}, \mathbf{x}_{i}^{\prime}, t\right) .
$$

The focusing function $f_{1}\left(\mathbf{x}, \mathbf{x}_{i}^{\prime}, t\right)$ is defined such that it focuses at $\mathbf{x}_{\mathrm{H}}=\mathbf{x}_{\mathrm{H}}^{\prime}$ at depth level $\partial \mathbb{D}_{i}$ and continues as a diverging downgoing field $f_{1}^{+}\left(\mathbf{x}, \mathbf{x}_{i}^{\prime}, t\right)$ into the reflection-free reference half-space $x_{3} \geq x_{3, i}$. Formally, the focusing condition is defined as (analogous to equation 3 )

$$
\left.\partial_{3} f_{1}^{+}\left(\mathbf{x}, \mathbf{x}_{i}^{\prime}, t\right)\right|_{x_{3}=x_{3, i}}=-\frac{1}{2} \rho\left(\mathbf{x}_{i}^{\prime}\right) \delta\left(\mathbf{x}_{\mathrm{H}}-\mathbf{x}_{\mathrm{H}}^{\prime}\right) \frac{\partial \delta(t)}{\partial t} .
$$

Similarly, the focusing function $f_{2}\left(\mathbf{x}, \mathbf{x}_{0}^{\prime \prime}, t\right)$ in Figure $2 \mathrm{~b}$ is defined as a superposition of downgoing and upgoing components, according to

$$
f_{2}\left(\mathbf{x}, \mathbf{x}_{0}^{\prime \prime}, t\right)=f_{2}^{+}\left(\mathbf{x}, \mathbf{x}_{0}^{\prime \prime}, t\right)+f_{2}^{-}\left(\mathbf{x}, \mathbf{x}_{0}^{\prime \prime}, t\right) .
$$

It focuses at $\mathbf{x}_{\mathrm{H}}=\mathbf{x}_{\mathrm{H}}^{\prime \prime}$ at depth level $\partial \mathbb{D}_{0}$ and continues as a diverging upgoing field $f_{2}^{-}\left(\mathbf{x}, \mathbf{x}_{0}^{\prime \prime}, t\right)$ into the homogeneous half-space $x_{3} \leq x_{3,0}$. This condition is formally defined as

$$
\left.\partial_{3} f_{2}^{-}\left(\mathbf{x}, \mathbf{x}_{0}^{\prime \prime}, t\right)\right|_{x_{3}=x_{3,0}}=\frac{1}{2} \rho\left(\mathbf{x}_{0}^{\prime \prime}\right) \delta\left(\mathbf{x}_{\mathrm{H}}-\mathbf{x}_{\mathrm{H}}^{\prime \prime}\right) \frac{\partial \delta(t)}{\partial t} .
$$

To avoid unstable behavior of the focusing functions, evanescent waves are excluded. This implies that the spatial delta functions in equations 6 and 8 should be interpreted in a band-limited sense, see also the example in the next section. This band-limitation of the focusing function is the same as that of standard migration schemes. It imposes a limit to the maximum obtainable spatial resolution (Berkhout and van Wulfften Palthe, 1979).

\section{Example of a focusing function}

To provide some insight into the properties of the focusing functions, here we present a somewhat intuitive preview of the focusing function $f_{1}^{+}\left(\mathbf{x}, \mathbf{x}_{i}^{\prime}, t\right)$ (the derivation is discussed later). Figure $3 \mathrm{a}$ shows a 2D inhomogeneous subsurface model (the different colors represent different propagation velocities). The red rays represent a wavefield emitted from the surface $\partial \mathbb{D}_{0}$ into the subsurface, with the aim to focus at $\mathbf{x}_{i}^{\prime}$ at $\partial \mathbb{D}_{i}$ and at $t=0$. The initial estimate of this focusing wavefield at $\partial \mathbb{D}_{0}, f_{1}^{+}\left(\mathbf{x}_{0}, \mathbf{x}_{i}^{\prime}, t\right)$, is obtained by time-reverting the response at $\partial \mathbb{D}_{0}$ to a point source at $\mathbf{x}_{i}^{\prime}$. It is shown in graylevel display in Figure $3 \mathrm{~b}$. We let this initial estimate propagate through the medium and evaluate its response at $\partial \mathbb{D}_{i}$. Its vertical derivative at $\partial \mathbb{D}_{i}$ (conform equation 6) is shown in Figure 3c. We observe a focus at the lateral position of the point source at $t=$ 0 and many events at positive times. Note that the display in Figure $3 \mathrm{c}$ is clipped to enhance these events; as a consequence, the artifacts of the focus around $t=0$ are also enhanced. The events at positive times are a result of multiple reflections, see Figure $3 \mathrm{~d}$. These extra arrivals violate the focusing condition of equation 6 , which states that $\partial_{3} f_{1}^{+}\left(\mathbf{x}, \mathbf{x}_{i}^{\prime}, t\right)$ evaluated at $\partial \mathbb{D}_{i}$ should reveal a focus only. Figure 4a shows a ray diagram (in red) of the actual focusing wavefield $f_{1}^{+}\left(\mathbf{x}, \mathbf{x}_{i}^{\prime}, t\right)$. The blue rays represent the reflected field $f_{1}^{-}\left(\mathbf{x}, \mathbf{x}_{i}^{\prime}, t\right)$. The additional red rays reach the interfaces at the same time as the upgoing blue rays, in such a way that they compensate for the downward reflected red rays in Figure 3d. Figure 4b shows the actual focusing wavefield at $\partial \mathbb{D}_{0}$ and Figure $4 \mathrm{c}$ the vertical derivative of its response at $\partial \mathbb{D}_{i}$. Note that in the latter figure, there are no undesired events at positive times; only the focus

a)

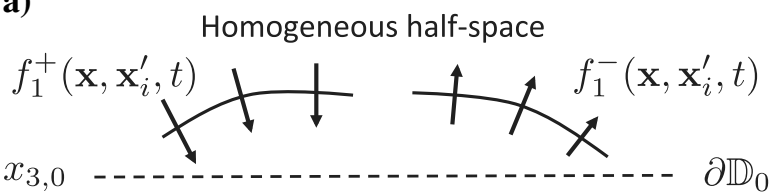

Actual inhomogeneous medium

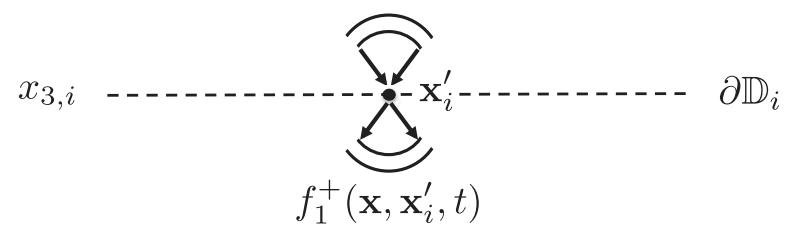

Reflection-free reference half-space

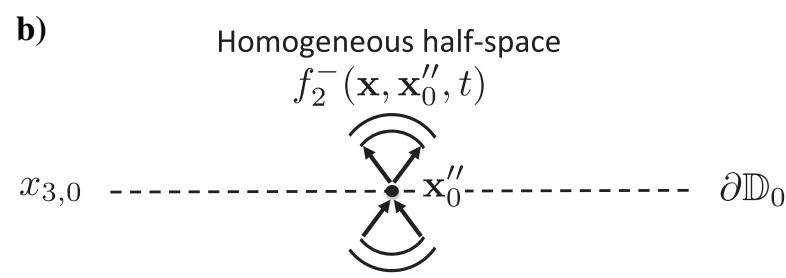

Actual inhomogeneous medium

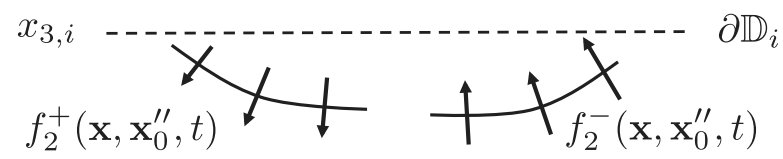

Reflection-free reference half-space

Figure 2. Downgoing and upgoing components of the focusing functions of the $3 \mathrm{D}$ wave equation in a reference configuration. (a) The function $f_{1}\left(\mathbf{x}, \mathbf{x}_{i}^{\prime}, t\right)$. (b) The function $f_{2}\left(\mathbf{x}, \mathbf{x}_{0}^{\prime \prime}, t\right)$. 
remains. The same clipping factor has been applied as in Figure 3c; hence, the focusing artifacts are again enhanced. The inset in Figure $4 \mathrm{c}$ shows a cross section of the focus at the central frequency $(20 \mathrm{~Hz})$. It is approximately a sinc-function, with the zero-crossings of the main lobe separated $125 \mathrm{~m}$, which is close to $\lambda / \sin \alpha_{\max }$, where $\lambda$ is the wavelength $(2300 / 20=115 \mathrm{~m})$ and $\alpha_{\max }$ the maximum propagation angle $\left(64^{\circ}\right)$. This confirms that the focus repre- sents a spatially band-limited delta-function (resulting from the absence of evanescent waves and large angles of incidence). We emphasize that, although in this example, we propagated the focusing function through the exact subsurface model to evaluate its focusing properties, we do not need this exact model to estimate the focusing function. We show later that the focusing functions can be derived from the measured reflection response at the surface and an estimate of the direct arrivals between the focus position and the receivers at the surface. For the estimation of the direct arrivals, a smooth subsurface model suffices.

\section{Relations between Green's functions and focusing functions}

At depth levels $\partial \mathbb{D}_{0}$ and $\partial \mathbb{D}_{i}$, the one-way focusing functions are mutually related, according to

$$
f_{1}^{+}\left(\mathbf{x}_{0}^{\prime \prime}, \mathbf{x}_{i}^{\prime}, t\right)=f_{2}^{-}\left(\mathbf{x}_{i}^{\prime}, \mathbf{x}_{0}^{\prime \prime}, t\right)
$$

and

$$
-f_{1}^{-}\left(\mathbf{x}_{0}^{\prime \prime}, \mathbf{x}_{i}^{\prime},-t\right)=f_{2}^{+}\left(\mathbf{x}_{i}^{\prime}, \mathbf{x}_{0}^{\prime \prime}, t\right),
$$

respectively (Appendix A, equations A-9 and A10). The one-way Green's functions at depth level $\partial \mathbb{D}_{i}$ (Figure 1), the focusing functions $f_{1}^{ \pm}$ at depth level $\partial \mathbb{D}_{0}$ (Figure $2 \mathrm{a}$ ), and the reflection response at depth level $\partial \mathbb{D}_{0}$ are mutually related via

$$
\begin{aligned}
& G^{-}\left(\mathbf{x}_{i}^{\prime}, \mathbf{x}_{0}^{\prime \prime}, t\right)= \\
& \quad \int_{\partial \mathbb{D}_{0}} \mathrm{~d} \mathbf{x}_{0} \int_{-\infty}^{t} R^{\cup}\left(\mathbf{x}_{0}^{\prime \prime}, \mathbf{x}_{0}, t-t^{\prime}\right) f_{1}^{+}\left(\mathbf{x}_{0}, \mathbf{x}_{i}^{\prime}, t^{\prime}\right) \mathrm{d} t^{\prime} \\
& -f_{1}^{-}\left(\mathbf{x}_{0}^{\prime \prime}, \mathbf{x}_{i}^{\prime}, t\right)
\end{aligned}
$$

and a)

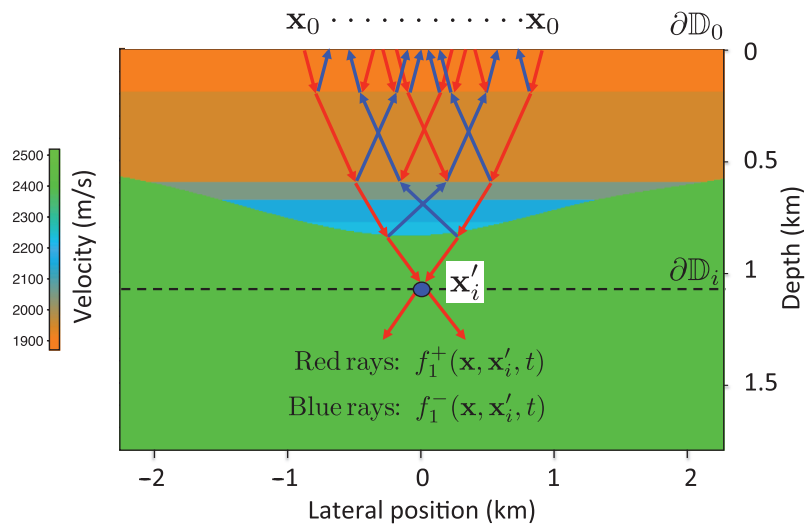

b)

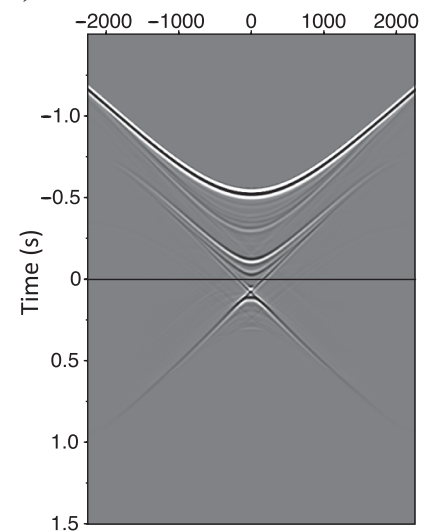

c) Lateral position $(\mathrm{km})$

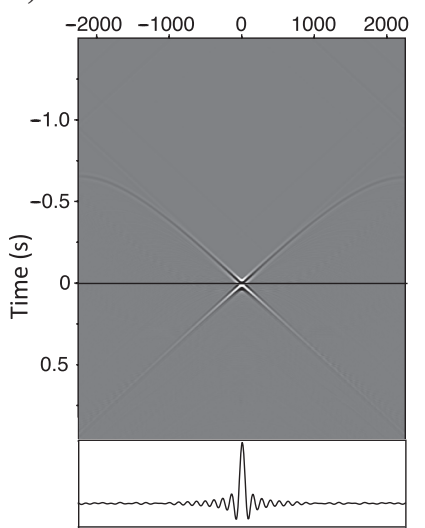

Figure 4. Illustration of the actual focusing function $f_{1}^{+}\left(\mathbf{x}, \mathbf{x}_{i}^{\prime}, t\right)$. (a) Ray diagram of the actual focusing function. (b) Actual focusing function at $\partial \mathbb{D}_{0}$. (c) Its response at $\partial \mathbb{D}_{i}$. Inset: cross section of the focus at the central frequency. 


$$
\begin{aligned}
& G^{+}\left(\mathbf{x}_{i}^{\prime}, \mathbf{x}_{0}^{\prime \prime}, t\right)= \\
& \quad-\int_{\partial \mathbb{D}_{0}} \mathrm{~d} \mathbf{x}_{0} \int_{-\infty}^{t} R^{\cup}\left(\mathbf{x}_{0}^{\prime \prime}, \mathbf{x}_{0}, t-t^{\prime}\right) f_{1}^{-}\left(\mathbf{x}_{0}, \mathbf{x}_{i}^{\prime},-t^{\prime}\right) \mathrm{d} t^{\prime} \\
& \quad+f_{1}^{+}\left(\mathbf{x}_{0}^{\prime \prime}, \mathbf{x}_{i}^{\prime},-t\right)
\end{aligned}
$$

see Appendix A, equations A-11 and A-12. The upper integration limit of the time integral $\left(t^{\prime}=t\right)$ follows from the causality of the reflection response. Adding the left- and right sides of these expressions, using equations $2,7,9$, and 10 , we obtain

$$
\begin{aligned}
& G\left(\mathbf{x}_{i}^{\prime}, \mathbf{x}_{0}^{\prime \prime}, t\right)= \\
& \quad \int_{\partial \mathbb{D}_{0}} \mathrm{~d} \mathbf{x}_{0} \int_{-\infty}^{t} R^{\cup}\left(\mathbf{x}_{0}^{\prime \prime}, \mathbf{x}_{0}, t-t^{\prime}\right) f_{2}\left(\mathbf{x}_{i}^{\prime}, \mathbf{x}_{0}, t^{\prime}\right) \mathrm{d} t^{\prime} \\
& \quad+f_{2}\left(\mathbf{x}_{i}^{\prime}, \mathbf{x}_{0}^{\prime \prime},-t\right)
\end{aligned}
$$

This equation relates the Green's function $G\left(\mathbf{x}_{i}^{\prime}, \mathbf{x}_{0}^{\prime \prime}, t\right)$, with $\mathbf{x}_{i}^{\prime}$ in the subsurface, to the reflection response $R^{\cup}\left(\mathbf{x}_{0}^{\prime \prime}, \mathbf{x}_{0}, t\right)$, measured at the surface, and the focusing function $f_{2}\left(\mathbf{x}_{i}^{\prime}, \mathbf{x}_{0}^{\prime \prime}, t\right)$. In the next section, we show that $f_{2}\left(\mathbf{x}_{i}^{\prime}, \mathbf{x}_{0}^{\prime \prime}, t\right)$ can be derived from the reflection response and the direct arrival $G_{\mathrm{d}}\left(\mathbf{x}_{i}^{\prime}, \mathbf{x}_{0}^{\prime \prime}, t\right)$ of the Green's function. Together with equation 13, this implies that the full Green's function $G\left(\mathbf{x}_{i}^{\prime}, \mathbf{x}_{0}^{\prime \prime}, t\right)$ is retrieved from its direct arrival and the reflection response at the surface.

\section{GREEN'S FUNCTION RETRIEVAL}

\section{Marchenko equation}

Equation 13 is the starting point for deriving the 3D Marchenko equation. Because of causality, the left side is zero before the first arrival of the Green's function. Hence,

$$
\begin{aligned}
0= & \int_{\partial \mathbb{D}_{0}} \mathrm{~d} \mathbf{x}_{0} \int_{-\infty}^{t} R^{\cup}\left(\mathbf{x}_{0}^{\prime \prime}, \mathbf{x}_{0}, t-t^{\prime}\right) f_{2}\left(\mathbf{x}_{i}^{\prime}, \mathbf{x}_{0}, t^{\prime}\right) \mathrm{d} t^{\prime} \\
& +f_{2}\left(\mathbf{x}_{i}^{\prime}, \mathbf{x}_{0}^{\prime \prime},-t\right), \quad \text { for } \quad t<t_{\mathrm{d}}\left(\mathbf{x}_{i}^{\prime}, \mathbf{x}_{0}^{\prime \prime}\right),
\end{aligned}
$$

where $t_{\mathrm{d}}\left(\mathbf{x}_{i}^{\prime}, \mathbf{x}_{0}^{\prime \prime}\right)$ denotes the traveltime of the first arrival of $G\left(\mathbf{x}_{i}^{\prime}, \mathbf{x}_{0}^{\prime \prime}, t\right)$ (in case of a triplicated wave, $t_{\mathrm{d}}\left(\mathbf{x}_{i}^{\prime}, \mathbf{x}_{0}^{\prime \prime}\right)$ is the traveltime of the first onset). By convolving equation 14 with an arbitrary symmetric function $h(t)$, it follows that when $f_{2}$ is a solution, then so is the convolution of $f_{2}$ with $h(t)$. This means that this equation has no unique solution for $f_{2}$. To constrain the solution, we assume that $f_{2}\left(\mathbf{x}_{i}^{\prime}, \mathbf{x}_{0}^{\prime \prime}, t\right)$ can be written as a direct wave followed by a scattering coda, analogous to the 1D case (Lamb, 1980). If the direct wave is known, then solving equation 14 reduces to solving the scattering coda. Before we define the direct wave of $f_{2}\left(\mathbf{x}_{i}^{\prime}, \mathbf{x}_{0}^{\prime \prime}, t\right)$, we first note that its upgoing part equals the inverse of the transmission response of the medium between $\partial \mathbb{D}_{0}$ and $\partial \mathbb{D}_{i}$, according to

$$
f_{2}^{-}\left(\mathbf{x}_{i}^{\prime}, \mathbf{x}_{0}^{\prime \prime}, t\right)=\mathcal{T}^{\text {inv }}\left(\mathbf{x}_{i}^{\prime}, \mathbf{x}_{0}^{\prime \prime}, t\right),
$$

see Appendix A, in particular equation A-14. Because $f_{2}^{-}\left(\mathbf{x}_{i}^{\prime}, \mathbf{x}_{0}^{\prime \prime}, t\right)$ is the field that is incident to the reference configuration (see Figure $2 \mathrm{~b}$ ), its direct wave is also the direct wave of $f_{2}\left(\mathbf{x}_{i}^{\prime}, \mathbf{x}_{0}^{\prime \prime}, t\right)$. Therefore, we write

$$
f_{2}\left(\mathbf{x}_{i}^{\prime}, \mathbf{x}_{0}^{\prime \prime}, t\right)=\mathcal{T}_{\mathrm{d}}^{\mathrm{inv}}\left(\mathbf{x}_{i}^{\prime}, \mathbf{x}_{0}^{\prime \prime}, t\right)+M\left(\mathbf{x}_{i}^{\prime}, \mathbf{x}_{0}^{\prime \prime}, t\right),
$$

where $\mathcal{T}_{\mathrm{d}}^{\text {inv }}\left(\mathbf{x}_{i}^{\prime}, \mathbf{x}_{0}^{\prime \prime}, t\right)$ is the direct arrival of the inverse of the transmission response (including possible triplications due to multipathing). Its arrival time is $-t_{\mathrm{d}}\left(\mathbf{x}_{i}^{\prime}, \mathbf{x}_{0}^{\prime \prime}\right)$ (possible triplications in $\mathcal{T}_{\mathrm{d}}^{\text {inv }}\left(\mathbf{x}_{i}^{\prime}, \mathbf{x}_{0}^{\prime \prime}, t\right)$ arrive before this time $) . M\left(\mathbf{x}_{i}^{\prime}, \mathbf{x}_{0}^{\prime \prime}, t\right)$ is the scattering coda that follows the direct arrival, with

$$
M\left(\mathbf{x}_{i}^{\prime}, \mathbf{x}_{0}^{\prime \prime}, t\right)=0, \quad \text { for } t \leq-t_{\mathrm{d}}\left(\mathbf{x}_{i}^{\prime}, \mathbf{x}_{0}^{\prime \prime}\right) .
$$

The transmission response $\mathcal{T}\left(\mathbf{x}_{i}^{\prime}, \mathbf{x}_{0}^{\prime \prime}, t\right)$ is related in a specific way to the Green's function of the medium between $\partial \mathbb{D}_{0}$ and $\partial \mathbb{D}_{i}$ (see equations A-13 and A-15 in Appendix A). It is often sufficient to approximate the direct arrival of its inverse by

$$
\mathcal{T}_{\mathrm{d}}^{\text {inv }}\left(\mathbf{x}_{i}^{\prime}, \mathbf{x}_{0}^{\prime \prime}, t\right) \approx G_{\mathrm{d}}\left(\mathbf{x}_{i}^{\prime}, \mathbf{x}_{0}^{\prime \prime},-t\right),
$$

where $G_{\mathrm{d}}\left(\mathbf{x}_{i}^{\prime}, \mathbf{x}_{0}^{\prime \prime},-t\right)$ is the time reversal of the direct arrival of the Green's function (including possible triplications). This approximation mainly implies that transmission losses at the interfaces are ignored (Wapenaar and Berkhout, 1989). In the following derivation, we continue with $\mathcal{T}_{\mathrm{d}}^{\text {inv }}\left(\mathbf{x}_{i}^{\prime}, \mathbf{x}_{0}^{\prime \prime}, t\right)$, but in the numerical examples, we will approximate it by $G_{\mathrm{d}}\left(\mathbf{x}_{i}^{\prime}, \mathbf{x}_{0}^{\prime \prime},-t\right)$.

By expressing $f_{2}$ as a direct wave, followed by a causal scattering coda (equations 16 and 17), we tacitly assumed that the first arriving wave is the direct wave. In the following, we call this the "directwave assumption." This assumption is not always fulfilled. For example, at large horizontal distances, the first arriving wave may be a refracted wave. The conditions for the direct-wave assumption need further investigation. The following analysis is limited to situations for which this assumption is valid, for example, for finite horizontal distances in layered media with moderately curved interfaces.

Substituting equation 16 into 14 , using the causality condition 17, yields the 3D Marchenko equation:

$$
\begin{aligned}
& \int_{\partial \mathbb{D}_{0}} \mathrm{~d} \mathbf{x}_{0} \int_{-\infty}^{-t_{\mathrm{d}}^{e}\left(\mathbf{x}_{i}^{\prime}, \mathbf{x}_{0}\right)} R^{\cup}\left(\mathbf{x}_{0}^{\prime \prime}, \mathbf{x}_{0}, t-t^{\prime}\right) \mathcal{T}_{\mathrm{d}}^{\mathrm{inv}}\left(\mathbf{x}_{i}^{\prime}, \mathbf{x}_{0}, t^{\prime}\right) \mathrm{d} t^{\prime} \\
& \quad+\int_{\partial \mathbb{D}_{0}} \mathrm{~d} \mathbf{x}_{0} \int_{-t_{\mathrm{d}}^{e}\left(\mathbf{x}_{i}^{\prime}, \mathbf{x}_{0}\right)}^{t} R^{\cup}\left(\mathbf{x}_{0}^{\prime \prime}, \mathbf{x}_{0}, t-t^{\prime}\right) M\left(\mathbf{x}_{i}^{\prime}, \mathbf{x}_{0}, t^{\prime}\right) \mathrm{d} t^{\prime} \\
& \quad+M\left(\mathbf{x}_{i}^{\prime}, \mathbf{x}_{0}^{\prime \prime},-t\right)=0, \text { for } t<t_{\mathrm{d}}\left(\mathbf{x}_{i}^{\prime}, \mathbf{x}_{0}^{\prime \prime}\right)
\end{aligned}
$$

with $t_{\mathrm{d}}^{\epsilon}\left(\mathbf{x}_{i}^{\prime}, \mathbf{x}_{0}\right)=t_{\mathrm{d}}\left(\mathbf{x}_{i}^{\prime}, \mathbf{x}_{0}\right)-\epsilon$, where $\epsilon$ is a small positive constant (introduced so that the direct arrival is included in the first integral). The Marchenko equation is solved for $M\left(\mathbf{x}_{i}^{\prime}, \mathbf{x}_{0}^{\prime \prime}, t\right)$ by the following iterative scheme:

$$
\begin{aligned}
& M_{k}\left(\mathbf{x}_{i}^{\prime}, \mathbf{x}_{0}^{\prime \prime},-t\right)=M_{0}\left(\mathbf{x}_{i}^{\prime}, \mathbf{x}_{0}^{\prime \prime},-t\right) \\
& \quad-\int_{\partial \mathbb{D}_{0}} \mathrm{~d} \mathbf{x}_{0} \int_{-t_{\mathrm{d}}^{\epsilon}\left(\mathbf{x}_{i}^{\prime}, \mathbf{x}_{0}\right)}^{t} R^{\cup}\left(\mathbf{x}_{0}^{\prime \prime}, \mathbf{x}_{0}, t-t^{\prime}\right) M_{k-1}\left(\mathbf{x}_{i}^{\prime}, \mathbf{x}_{0}, t^{\prime}\right) \mathrm{d} t^{\prime},
\end{aligned}
$$

with

$$
\begin{aligned}
& M_{0}\left(\mathbf{x}_{i}^{\prime}, \mathbf{x}_{0}^{\prime \prime},-t\right)= \\
& -\int_{\partial \mathbb{D}_{0}} \mathrm{~d} \mathbf{x}_{0} \int_{-\infty}^{-t_{\mathrm{d}}^{e}\left(\mathbf{x}_{i}^{\prime}, \mathbf{x}_{0}\right)} R^{\cup}\left(\mathbf{x}_{0}^{\prime \prime}, \mathbf{x}_{0}, t-t^{\prime}\right) \mathcal{T}_{\mathrm{d}}^{\text {inv }}\left(\mathbf{x}_{i}^{\prime}, \mathbf{x}_{0}, t^{\prime}\right) \mathrm{d} t^{\prime},
\end{aligned}
$$


for $t<t_{\mathrm{d}}\left(\mathbf{x}_{i}^{\prime}, \mathbf{x}_{0}^{\prime \prime}\right)$, whereas $M_{k}\left(\mathbf{x}_{i}^{\prime}, \mathbf{x}_{0}^{\prime \prime},-t\right)=0$ for $t \geq t_{\mathrm{d}}\left(\mathbf{x}_{i}^{\prime}, \mathbf{x}_{0}^{\prime \prime}\right)$. Hence, assuming the reflection response at the surface and the direct arrival of the inverse transmission response is known, the $k$ th iteration of the coda, $M_{k}\left(\mathbf{x}_{i}^{\prime}, \mathbf{x}_{0}^{\prime \prime}, t\right)$, follows from equations 20 and 21 . Using equation 16 , the successive iterations for the focusing function $f_{2}\left(\mathbf{x}_{i}^{\prime}, \mathbf{x}_{0}^{\prime \prime}, t\right)$ are then given by

$$
f_{2, k}\left(\mathbf{x}_{i}^{\prime}, \mathbf{x}_{0}^{\prime \prime}, t\right)=\mathcal{T}_{\mathrm{d}}^{\text {inv }}\left(\mathbf{x}_{i}^{\prime}, \mathbf{x}_{0}^{\prime \prime}, t\right)+M_{k-1}\left(\mathbf{x}_{i}^{\prime}, \mathbf{x}_{0}^{\prime \prime}, t\right),
$$

with $M_{-1}\left(\mathbf{x}_{i}^{\prime}, \mathbf{x}_{0}^{\prime \prime}, t\right)=0$. Assuming the scheme converges, the Green's function $G\left(\mathbf{x}_{i}^{\prime}, \mathbf{x}_{0}^{\prime \prime}, t\right)$ is subsequently obtained by substituting the converged solution $f_{2}\left(\mathbf{x}_{i}^{\prime}, \mathbf{x}_{0}^{\prime \prime}, t\right)$ into equation 13. A numerical example of this scheme, for a situation with a triplicated direct wave, is discussed in Wapenaar et al. (2014). Here, we continue with decomposition before we discuss a numerical example.

\section{Decomposition of the Marchenko equation}

The Marchenko equation, discussed in the previous section, forms the basis for retrieving the two-way Green's function $G\left(\mathbf{x}_{i}^{\prime}, \mathbf{x}_{0}^{\prime \prime}, t\right)=G^{+}\left(\mathbf{x}_{i}^{\prime}, \mathbf{x}_{0}^{\prime \prime}, t\right)+G^{-}\left(\mathbf{x}_{i}^{\prime}, \mathbf{x}_{0}^{\prime \prime}, t\right)$ from the reflection response $R^{\cup}\left(\mathbf{x}_{0}^{\prime \prime}, \mathbf{x}_{0}, t\right)$ at the surface and the direct arrival of the inverse transmission response $\mathcal{T}_{\mathrm{d}}^{\text {inv }}\left(\mathbf{x}_{i}^{\prime}, \mathbf{x}_{0}^{\prime \prime}, t\right)$. For redatuming and reflection imaging, the one-way Green's functions $G^{-}\left(\mathbf{x}_{i}^{\prime}, \mathbf{x}_{0}^{\prime \prime}, t\right)$ and $G^{+}\left(\mathbf{x}_{i}^{\prime}, \mathbf{x}_{0}^{\prime \prime}, t\right)$ are needed separately. Different approaches to decompose the two-way Green's function into one-way Green's functions are possible (Wapenaar et al., 2012; Slob et al., 2014). We follow the approach of Slob et al. (2014), modified for the 3D situation. This approach uses the one-way Green's function representations 11 and 12 .

Because of causality, the left sides of equations 11 and 12 are zero before the first arrival of the Green's function. Hence,

$$
\begin{aligned}
0 & =\int_{\partial \mathbb{D}_{0}} \mathrm{~d} \mathbf{x}_{0} \int_{-\infty}^{t} R^{\cup}\left(\mathbf{x}_{0}^{\prime \prime}, \mathbf{x}_{0}, t-t^{\prime}\right) f_{1}^{+}\left(\mathbf{x}_{0}, \mathbf{x}_{i}^{\prime}, t^{\prime}\right) \mathrm{d} t^{\prime} \\
& -f_{1}^{-}\left(\mathbf{x}_{0}^{\prime \prime}, \mathbf{x}_{i}^{\prime}, t\right) \text { for } t<t_{\mathrm{d}}\left(\mathbf{x}_{i}^{\prime}, \mathbf{x}_{0}^{\prime \prime}\right)
\end{aligned}
$$

and

$$
\begin{aligned}
0= & \int_{\partial \mathbb{D}_{0}} \mathrm{~d} \mathbf{x}_{0} \int_{-\infty}^{t} R^{\cup}\left(\mathbf{x}_{0}^{\prime \prime}, \mathbf{x}_{0}, t-t^{\prime}\right) f_{1}^{-}\left(\mathbf{x}_{0}, \mathbf{x}_{i}^{\prime},-t^{\prime}\right) \mathrm{d} t^{\prime} \\
& -f_{1}^{+}\left(\mathbf{x}_{0}^{\prime \prime}, \mathbf{x}_{i}^{\prime},-t\right) \text { for } t<t_{\mathrm{d}}\left(\mathbf{x}_{i}^{\prime}, \mathbf{x}_{0}^{\prime \prime}\right) .
\end{aligned}
$$

Analogous to equations 16 and $17, f_{1}^{+}\left(\mathbf{x}_{0}^{\prime \prime}, \mathbf{x}_{i}^{\prime}, t\right)$ is written as

$$
f_{1}^{+}\left(\mathbf{x}_{0}^{\prime \prime}, \mathbf{x}_{i}^{\prime}, t\right)=\mathcal{T}_{\mathrm{d}}^{\text {inv }}\left(\mathbf{x}_{i}^{\prime}, \mathbf{x}_{0}^{\prime \prime}, t\right)+M^{+}\left(\mathbf{x}_{0}^{\prime \prime}, \mathbf{x}_{i}^{\prime}, t\right),
$$

where $M^{+}\left(\mathbf{x}_{0}^{\prime \prime}, \mathbf{x}_{i}^{\prime}, t\right)$ is a causal coda, with

$$
M^{+}\left(\mathbf{x}_{0}^{\prime \prime}, \mathbf{x}_{i}^{\prime}, t\right)=0, \quad \text { for } t \leq-t_{\mathrm{d}}\left(\mathbf{x}_{i}^{\prime}, \mathbf{x}_{0}^{\prime \prime}\right) .
$$

For $f_{1}^{-}\left(\mathbf{x}_{0}^{\prime \prime}, \mathbf{x}_{i}^{\prime}, t\right)$, we obtain from equation 10 and the causality of $f_{2}\left(\mathbf{x}_{i}^{\prime}, \mathbf{x}_{0}^{\prime \prime}, t\right)$ :

$$
f_{1}^{-}\left(\mathbf{x}_{0}^{\prime \prime}, \mathbf{x}_{i}^{\prime}, t\right)=0, \quad \text { for } t \geq t_{\mathrm{d}}\left(\mathbf{x}_{i}^{\prime}, \mathbf{x}_{0}^{\prime \prime}\right) .
$$

As in the previous section, the following analysis only applies to situations for which the "direct-wave assumption" (equations 2527) holds true.
Substituting equation 25 into equations 23 and 24, using the causality conditions 26 and 27 , gives

$$
\begin{aligned}
& \int_{\partial \mathbb{D}_{0}} \mathrm{~d} \mathbf{x}_{0} \int_{-\infty}^{-t_{\mathrm{d}}^{\epsilon}\left(\mathbf{x}_{i}^{\prime}, \mathbf{x}_{0}\right)} R^{\cup}\left(\mathbf{x}_{0}^{\prime \prime}, \mathbf{x}_{0}, t-t^{\prime}\right) \mathcal{T}_{\mathrm{d}}^{\mathrm{inv}}\left(\mathbf{x}_{i}^{\prime}, \mathbf{x}_{0}, t^{\prime}\right) \mathrm{d} t^{\prime} \\
& +\int_{\partial \mathbb{D}_{0}} \mathrm{~d} \mathbf{x}_{0} \int_{-t_{\mathrm{d}}^{\epsilon}\left(\mathbf{x}_{i}^{\prime}, \mathbf{x}_{0}\right)}^{t} R^{\cup}\left(\mathbf{x}_{0}^{\prime \prime}, \mathbf{x}_{0}, t-t^{\prime}\right) M^{+}\left(\mathbf{x}_{0}, \mathbf{x}_{i}^{\prime}, t^{\prime}\right) \mathrm{d} t^{\prime} \\
& -f_{1}^{-}\left(\mathbf{x}_{0}^{\prime \prime}, \mathbf{x}_{i}^{\prime}, t\right)=0, \quad \text { for } t<t_{\mathrm{d}}\left(\mathbf{x}_{i}^{\prime}, \mathbf{x}_{0}^{\prime \prime}\right)
\end{aligned}
$$

and

$$
\begin{gathered}
\int_{\partial \mathbb{D}_{0}} \mathrm{~d} \mathbf{x}_{0} \int_{-t_{\mathrm{d}}^{e}\left(\mathbf{x}_{i}^{\prime}, \mathbf{x}_{0}\right)}^{t} R^{\cup}\left(\mathbf{x}_{0}^{\prime \prime}, \mathbf{x}_{0}, t-t^{\prime}\right) f_{1}^{-}\left(\mathbf{x}_{0}, \mathbf{x}_{i}^{\prime},-t^{\prime}\right) \mathrm{d} t^{\prime} \\
-M^{+}\left(\mathbf{x}_{0}^{\prime \prime}, \mathbf{x}_{i}^{\prime},-t\right)=0, \quad \text { for } \quad t<t_{\mathrm{d}}\left(\mathbf{x}_{i}^{\prime}, \mathbf{x}_{0}^{\prime \prime}\right) .
\end{gathered}
$$

This coupled system of Marchenko equations is solved by the following iterative scheme:

$$
\begin{aligned}
& M_{k}^{+}\left(\mathbf{x}_{0}^{\prime \prime}, \mathbf{x}_{i}^{\prime},-t\right)= \\
& \quad \int_{\partial \mathbb{D}_{0}} \mathrm{~d} \mathbf{x}_{0} \int_{-t_{\mathrm{d}}^{e}\left(\mathbf{x}_{i}^{\prime}, \mathbf{x}_{0}\right)}^{t} R^{\cup}\left(\mathbf{x}_{0}^{\prime \prime}, \mathbf{x}_{0}, t-t^{\prime}\right) f_{1, k}^{-}\left(\mathbf{x}_{0}, \mathbf{x}_{i}^{\prime},-t^{\prime}\right) \mathrm{d} t^{\prime}
\end{aligned}
$$

and

$$
\begin{aligned}
& f_{1, k+1}^{-}\left(\mathbf{x}_{0}^{\prime \prime}, \mathbf{x}_{i}^{\prime}, t\right)=f_{1,0}^{-}\left(\mathbf{x}_{0}^{\prime \prime}, \mathbf{x}_{i}^{\prime}, t\right) \\
& \quad+\int_{\partial \mathbb{D}_{0}} \mathrm{~d} \mathbf{x}_{0} \int_{-t_{\mathrm{d}}^{\epsilon}\left(\mathbf{x}_{i}^{\prime}, \mathbf{x}_{0}\right)}^{t} R^{\cup}\left(\mathbf{x}_{0}^{\prime \prime}, \mathbf{x}_{0}, t-t^{\prime}\right) M_{k}^{+}\left(\mathbf{x}_{0}, \mathbf{x}_{i}^{\prime}, t^{\prime}\right) \mathrm{d} t^{\prime},
\end{aligned}
$$

with

$$
\begin{aligned}
& f_{1,0}^{-}\left(\mathbf{x}_{0}^{\prime \prime}, \mathbf{x}_{i}^{\prime}, t\right)= \\
& \quad \int_{\partial \mathbb{D}_{0}} \mathrm{~d} \mathbf{x}_{0} \int_{-\infty}^{-t_{\mathrm{d}}^{\epsilon}\left(\mathbf{x}_{i}^{\prime}, \mathbf{x}_{0}\right)} R^{\cup}\left(\mathbf{x}_{0}^{\prime \prime}, \mathbf{x}_{0}, t-t^{\prime}\right) \mathcal{T}_{\mathrm{d}}^{\text {inv }}\left(\mathbf{x}_{i}^{\prime}, \mathbf{x}_{0}, t^{\prime}\right) \mathrm{d} t^{\prime},
\end{aligned}
$$

for $t<t_{\mathrm{d}}\left(\mathbf{x}_{i}^{\prime}, \mathbf{x}_{0}^{\prime \prime}\right)$, whereas $M_{k}^{+}\left(\mathbf{x}_{0}^{\prime \prime}, \mathbf{x}_{i}^{\prime},-t\right)=f_{1, k+1}^{-}\left(\mathbf{x}_{0}^{\prime \prime}, \mathbf{x}_{i}^{\prime}, t\right)=0$ for $t \geq t_{\mathrm{d}}\left(\mathbf{x}_{i}^{\prime}, \mathbf{x}_{0}^{\prime \prime}\right)$. Note that $f_{1,0}^{-}\left(\mathbf{x}_{0}^{\prime \prime}, \mathbf{x}_{i}^{\prime}, t\right)$, defined in equation 32, is identical to $-M_{0}\left(\mathbf{x}_{i}^{\prime}, \mathbf{x}_{0}^{\prime \prime},-t\right)$, defined in equation 21. In Appendix B, it is shown that $M_{k}^{+}\left(\mathbf{x}_{0}^{\prime \prime}, \mathbf{x}_{i}^{\prime}, t\right)$ and $f_{1, k}^{-}\left(\mathbf{x}_{0}^{\prime \prime}, \mathbf{x}_{i}^{\prime}, t\right)$ can alternatively be obtained from the odd and even terms of an expansion of the coda $M\left(\mathbf{x}_{i}^{\prime}, \mathbf{x}_{0}^{\prime \prime}, t\right)$ of the focusing function $f_{2}\left(\mathbf{x}_{i}^{\prime}, \mathbf{x}_{0}^{\prime \prime}, t\right)$.

Using equation 25 , the iterations of the one-way focusing function $f_{1}^{+}\left(\mathbf{x}_{0}^{\prime \prime}, \mathbf{x}_{i}^{\prime}, t\right)$ can be written as

$$
f_{1, k}^{+}\left(\mathbf{x}_{0}^{\prime \prime}, \mathbf{x}_{i}^{\prime}, t\right)=\mathcal{T}_{\mathrm{d}}^{\text {inv }}\left(\mathbf{x}_{i}^{\prime}, \mathbf{x}_{0}^{\prime \prime}, t\right)+M_{k-1}^{+}\left(\mathbf{x}_{0}^{\prime \prime}, \mathbf{x}_{i}^{\prime}, t\right),
$$

with $M_{-1}^{+}\left(\mathbf{x}_{0}^{\prime \prime}, \mathbf{x}_{i}^{\prime}, t\right)=0$. Assuming the scheme converges, the decomposed Green's functions $G^{-}\left(\mathbf{x}_{i}^{\prime}, \mathbf{x}_{0}^{\prime \prime}, t\right)$ and $G^{+}\left(\mathbf{x}_{i}^{\prime}, \mathbf{x}_{0}^{\prime \prime}, t\right)$ are obtained by substituting the converged solutions $f_{1}^{+}\left(\mathbf{x}_{0}^{\prime \prime}, \mathbf{x}_{i}^{\prime}, t\right)$ and $f_{1}^{-}\left(\mathbf{x}_{0}^{\prime \prime}, \mathbf{x}_{i}^{\prime}, t\right)$ into equations 11 and 12 .

\section{Numerical example of Green's function retrieval}

We illustrate the iterative solution of the one-way focusing functions, and the subsequent retrieval of the decomposed Green's 
functions, with a numerical example in a 2D inhomogeneous subsurface model (Figure 5). The propagation velocities in the different layers are represented by different colors in Figure 5a. The reflector package below $\partial \mathbb{D}_{i}$ (i.e., below $x_{3, i}=1100 \mathrm{~m}$ ) represents a target zone on which we will zoom in later. A smoothed version of the propagation velocity model is shown in Figure 5b. The numerically modeled reflection response $R^{\cup}\left(\mathbf{x}_{0}^{\prime \prime}, \mathbf{x}_{0}, t\right)$, convolved with a Ricker wavelet with a central frequency of $20 \mathrm{~Hz}$, is shown in gray-level display in Figure $5 \mathrm{c}$. The source position is fixed at $\mathbf{x}_{0}=(0,0)$ and the receiver position $\mathbf{x}_{0}^{\prime \prime}=\left(x_{1}^{\prime \prime}, 0\right)$ is variable, with $x_{1}^{\prime \prime}$ ranging from -2250 to $2250 \mathrm{~m}$, with an interreceiver distance of $10 \mathrm{~m}$. The upper half-space is homogeneous, so the reflection response contains no surface-related multiples. The response in Figure $5 \mathrm{c}$ is displayed with a small time-dependent gain of $\exp (0.1 * t)$ to emphasize the internal multiples. In total, 451 reflection responses $R^{\cup}\left(\mathbf{x}_{0}^{\prime \prime}, \mathbf{x}_{0}, t\right)$ have been modeled, for source positions $\mathbf{x}_{0}=\left(x_{1}, 0\right)$, with $x_{1}$ also ranging from -2250 to $2250 \mathrm{~m}$, with a source interval of $10 \mathrm{~m}$. Figure $5 \mathrm{~d}$ shows the direct arrival of the Green's function, $G_{\mathrm{d}}\left(\mathbf{x}_{i}^{\prime}, \mathbf{x}_{0}, t\right)$, for variable source position $\mathbf{x}_{0}$ (same range as above) and fixed receiver position $\mathbf{x}_{i}^{\prime}=(0,1100 \mathrm{~m})$. This Green's function has been modeled in a smoothed version of the model (Figure $5 \mathrm{~b}$ ), to acknowledge the fact that in practice, no precise information about the interfaces and medium parameters is available. The reflection response in Figure $5 \mathrm{c}$ and the direct arrival in Figure $5 \mathrm{~d}$ are input to the iterative scheme.

The iterative procedure starts with $f_{1,0}^{+}\left(\mathbf{x}_{0}\right.$, $\left.\mathbf{x}_{i}^{\prime}, t\right)=\mathcal{T}_{\mathrm{d}}^{\text {inv }}\left(\mathbf{x}_{i}^{\prime}, \mathbf{x}_{0}, t\right) \approx G_{\mathrm{d}}\left(\mathbf{x}_{i}^{\prime}, \mathbf{x}_{0},-t\right)$. This approximation mainly implies that we ignore transmission losses at the interfaces. This will affect the retrieved Green's functions $G^{-}\left(\mathbf{x}_{i}^{\prime}, \mathbf{x}_{0}^{\prime \prime}, t\right)$ and $G^{+}\left(\mathbf{x}_{i}^{\prime}, \mathbf{x}_{0}^{\prime \prime}, t\right)$ in approximately the same way. In the section "Marchenko redatuming," we will deconvolve $G^{+}\left(\mathbf{x}_{i}^{\prime}, \mathbf{x}_{0}^{\prime \prime}, t\right)$ with $G^{-}\left(\mathbf{x}_{i}^{\prime}\right.$, $\left.\mathbf{x}_{0}^{\prime \prime}, t\right)$. This deconvolution largely compensates for the mentioned approximation.

The zeroth order term of the focusing function, $f_{1,0}^{+}\left(\mathbf{x}_{0}, \mathbf{x}_{i}^{\prime}, t\right) \approx G_{\mathrm{d}}\left(\mathbf{x}_{i}^{\prime}, \mathbf{x}_{0},-t\right)$, is shown in Figure $6 \mathrm{a}$, for a fixed focal point $\mathbf{x}_{i}^{\prime}=$ $(0,1100 \mathrm{~m})$ and variable $\mathbf{x}_{0}=\left(x_{1}, 0\right)$. Its reflection response, $f_{1,0}^{-}\left(\mathbf{x}_{0}^{\prime \prime}, \mathbf{x}_{i}^{\prime}, t\right)$, is shown in Figure 6b. The functions $f_{1, k}^{+}\left(\mathbf{x}_{0}, \mathbf{x}_{i}^{\prime}, t\right)$ and $f_{1, k}^{-}\left(\mathbf{x}_{0}^{\prime \prime}, \mathbf{x}_{i}^{\prime}, t\right)$, for $k=1$ and $k=5$ are shown in Figure $6 \mathrm{c}-6 \mathrm{f}$. Note that new events are generated during iterations $k=0$ and $k=1$. During the higher order iterations, mainly the amplitudes of these events are modified. The results for $k=$ 1 (Figure 6c and 6d) are already very close to those for $k=5$ (Figure 6e and 6f).

The solutions after five iterations, shown in Figure 6e and 6f, are taken as the final estimates of $f_{1}^{+}\left(\mathbf{x}_{0}, \mathbf{x}_{i}^{\prime}, t\right)$ and $f_{1}^{-}\left(\mathbf{x}_{0}^{\prime \prime}, \mathbf{x}_{i}^{\prime}, t\right)$, respectively. These are used in equations 11 and 12 to retrieve the decomposed Green's functions $G^{-}\left(\mathbf{x}_{i}^{\prime}, \mathbf{x}_{0}^{\prime \prime}, t\right)$ and $G^{+}\left(\mathbf{x}_{i}^{\prime}, \mathbf{x}_{0}^{\prime \prime}, t\right)$. These are shown in Figure $7 \mathrm{a}$ and $7 \mathrm{~b}$, respectively, for a fixed virtual-receiver position $\mathbf{x}_{i}^{\prime}=(0$, $1100 \mathrm{~m})$ and variable source position $\mathbf{x}_{0}^{\prime \prime}$. They are displayed with a time-dependent gain of $\exp (1.0 * t)$ to emphasize the internal multiples. These decomposed Green's functions will be used in the section Marchenko redatuming. Figure 8 shows the sum of the results of Figure 7, displayed as seismic traces (only every 15th trace is shown). The black solid traces represent the retrieved Green's function, whereas the gray-dashed traces represent the directly modeled Green's function, as a reference (a time-dependent gain of $\exp (1.9 * t)$ has been applied). Note that the match is excellent, except for the far offsets of the first event.

\section{Physical interpretation}

We present a physical interpretation of the iterative scheme leading to the one-way focusing functions $f_{1}^{+}\left(\mathbf{x}_{0}, \mathbf{x}_{i}^{\prime}, t\right)$ and $f_{1}^{-}\left(\mathbf{x}_{0}^{\prime \prime}, \mathbf{x}_{i}^{\prime}, t\right)$, and of equations 11 and 12 for obtaining the decomposed Green's functions $G^{-}\left(\mathbf{x}_{i}^{\prime}, \mathbf{x}_{0}^{\prime \prime}, t\right)$ and $G^{+}\left(\mathbf{x}_{i}^{\prime}, \mathbf{x}_{0}^{\prime \prime}, t\right)$.

Figure 9 shows a physical interpretation of the first few iterations of the one-way focusing functions $f_{1, k}^{+}\left(\mathbf{x}_{0}, \mathbf{x}_{i}^{\prime}, t\right)$ and $f_{1, k}^{-}\left(\mathbf{x}_{0}^{\prime \prime}, \mathbf{x}_{i}^{\prime}, t\right)$. Recall that the focusing functions are defined in a reference configuration, which is equal to the actual medium above depth level $\partial \mathbb{D}_{i}$ and reflection-free below this depth level (see also Figure 2). Therefore, in Figure 9, the layers below $\partial \mathbb{D}_{i}$ are absent. The red rays in Figure 9a schematically represent the zeroth order term $f_{1,0}^{+}\left(\mathbf{x}_{0}, \mathbf{x}_{i}^{\prime}, t\right)=\mathcal{T}_{\mathrm{d}}^{\text {inv }}\left(\mathbf{x}_{i}^{\prime}, \mathbf{x}_{0}, t\right)$, which was shown in gray-level display in Figure 6 a (only two of 451 rays are shown). The rays start at

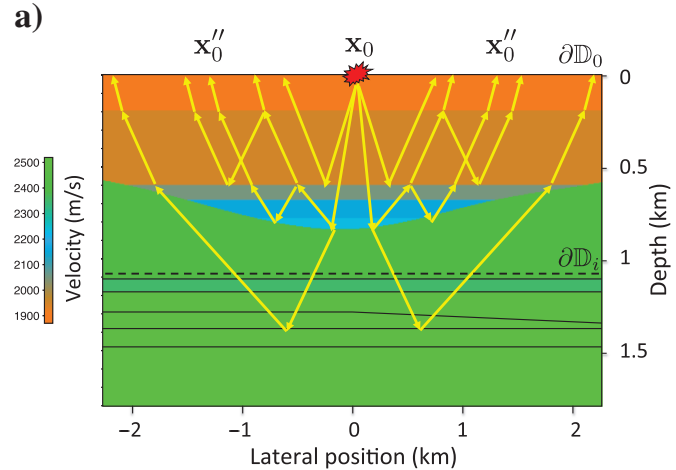

b)

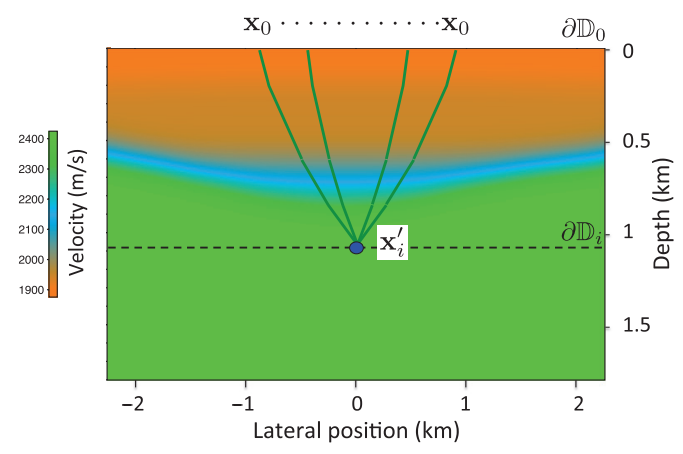

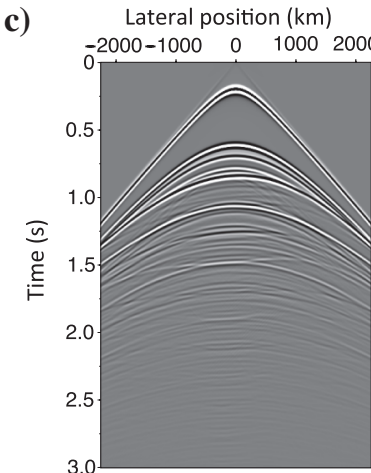

d)

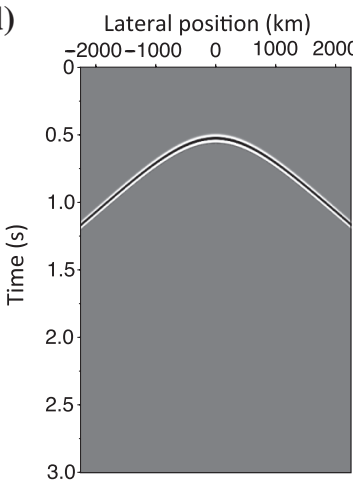

Figure 5. Two-dimensional inhomogeneous subsurface model. (a) Propagation velocity model, with some rays of the reflection response. (b) Smoothed version of the velocity model, with some rays of the direct arrival of the Green's function. (c) Reflection response $R^{\cup}\left(\mathbf{x}_{0}^{\prime \prime}, \mathbf{x}_{0}, t\right)$, for fixed $\mathbf{x}_{0}$ and variable $\mathbf{x}_{0}^{\prime \prime}$. (d) Direct arrival of the Green's function, $G_{\mathrm{d}}\left(\mathbf{x}_{i}^{\prime}, \mathbf{x}_{0}, t\right)$, for fixed $\mathbf{x}_{i}^{\prime}$ and variable $\mathbf{x}_{0}$. 
Wapenaar et al.

Figure 6 . The focusing functions $f_{1, k}^{+}\left(\mathbf{x}_{0}, \mathbf{x}_{i}^{\prime}, t\right)$ and $f_{1, k}^{-}\left(\mathbf{x}_{0}^{\prime \prime}, \mathbf{x}_{i}^{\prime}, t\right)$, for fixed $\mathbf{x}_{i}^{\prime}$ and variable $\mathbf{x}_{0}$ and $\mathbf{x}_{0}^{\prime \prime}$, for $k=0$ (Figure 6a and 6b), $k=1$ (Figure $6 \mathrm{c}$ and $6 \mathrm{~d}$ ), and $k=5$ (Figure $6 \mathrm{e}$ and $6 \mathrm{f}$ ).

a)

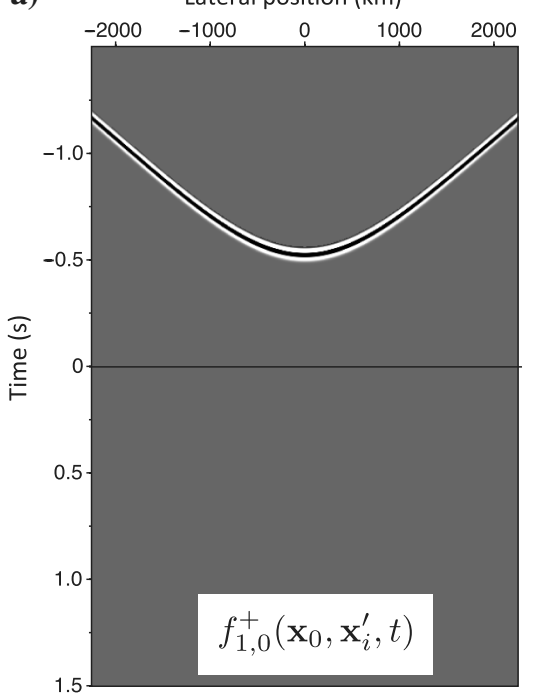

c)

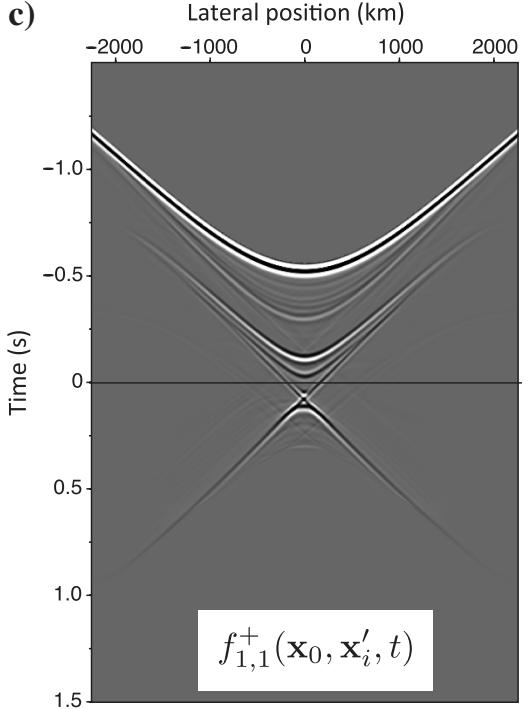

e)

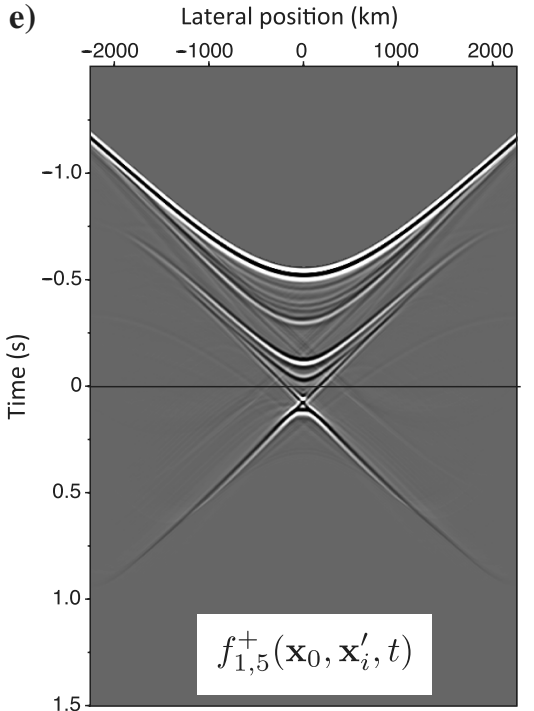

b) Lateral position $(\mathrm{km})$

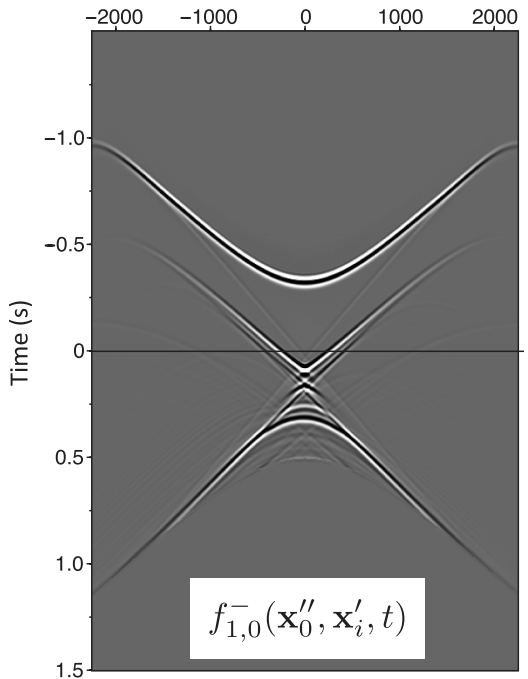

d)

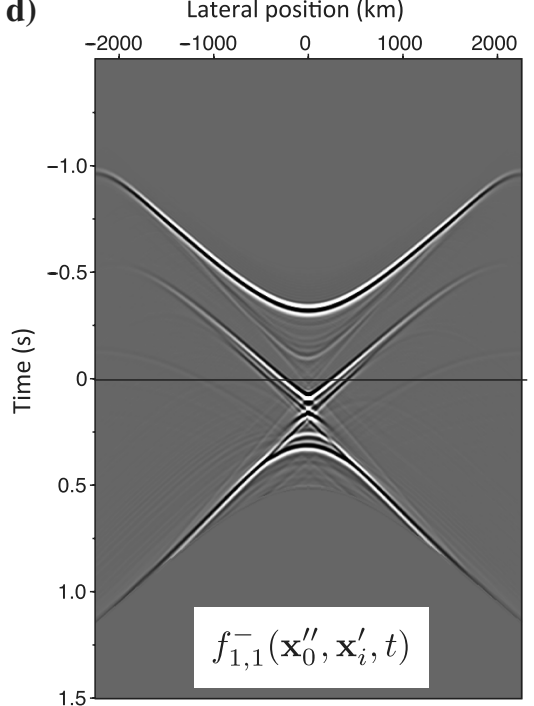

f)

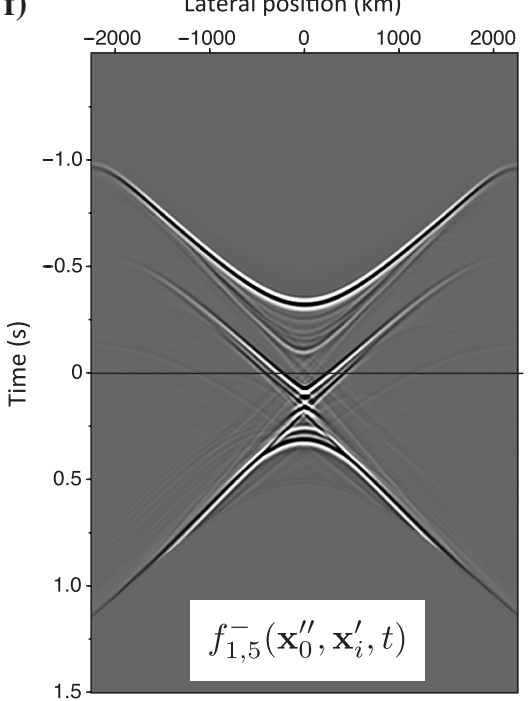


$\mathbf{x}_{0}=\left(x_{1}, 0\right)$ at the surface (for all $x_{1}$-values) and cross each other at the focal point $\mathbf{x}_{i}^{\prime}$ at $t=0$. During downward propagation toward the focal point, reflection occurs at the interfaces. This is expressed by equation 32 . The blue rays in Figure 9 a represent $f_{1,0}^{-}\left(\mathbf{x}_{0}^{\prime \prime}, \mathbf{x}_{i}^{\prime}, t\right)$. Although the integrand in equation 32 contains the reflection response of the actual medium, the result is evaluated only for $t<t_{\mathrm{d}}\left(\mathbf{x}_{i}^{\prime}, \mathbf{x}_{0}^{\prime \prime}\right)$, and therefore, reflections from below $\partial \mathbb{D}_{i}$ do not contribute. The blue rays correspond to the main events in $f_{1,0}^{-}\left(\mathbf{x}_{0}^{\prime \prime}, \mathbf{x}_{i}^{\prime}, t\right)$ in Figure $6 \mathrm{~b}$ (for simplicity not all reflections are shown in Figure 9a). Next, equation 30 is evaluated for $k=0$. Hence, the reflection response of the medium is applied to the time-reversed version of $f_{1,0}^{-}\left(\mathbf{x}_{0}, \mathbf{x}_{i}^{\prime}, t\right)$, which results in the time reversal of $M_{0}^{+}\left(\mathbf{x}_{0}^{\prime \prime}, \mathbf{x}_{i}^{\prime}, t\right)$. This is illustrated in Figure $9 \mathrm{~b}$. Here, the downgoing red rays are the reversals of the upgoing blue rays in Figure 9a. They represent $f_{1,0}^{-}\left(\mathbf{x}_{0}, \mathbf{x}_{i}^{\prime},-t\right)$, which is a downgoing field because of the time reversal. The upgoing blue rays in Figure $9 \mathrm{~b}$ represent $M_{0}^{+}\left(\mathbf{x}_{0}^{\prime \prime}, \mathbf{x}_{i}^{\prime},-t\right)$, which is an upgoing field because of the time reversal. Equation 30 is evaluated only for $t<t_{\mathrm{d}}\left(\mathbf{x}_{i}^{\prime}, \mathbf{x}_{0}^{\prime \prime}\right)$; hence, only the rays corresponding to arrivals for $t<t_{\mathrm{d}}\left(\mathbf{x}_{i}^{\prime}, \mathbf{x}_{0}^{\prime \prime}\right)$ are shown in Figure $9 \mathrm{~b}$. Next, $f_{1,1}^{+}\left(\mathbf{x}_{0}, \mathbf{x}_{i}^{\prime}, t\right)$ is constructed via $f_{1,1}^{+}\left(\mathbf{x}_{0}, \mathbf{x}_{i}^{\prime}, t\right)=$ $\mathcal{T}_{\mathrm{d}}^{\text {inv }}\left(\mathbf{x}_{i}^{\prime}, \mathbf{x}_{0}, t\right)+M_{0}^{+}\left(\mathbf{x}_{0}, \mathbf{x}_{i}^{\prime}, t\right)$. This is represented by the red rays in Figure 9c, consisting of the red rays in Figure 9a and the reversals of the blue rays in Figure 9b. Together, these red rays correspond to the main events in Figure $6 \mathrm{c}$ (the dashed parts of the red rays represent very weak events that vanish during the iterative process). Next, equation 31 is evaluated for $k=0$. This accomplishes that the reflection response to $M_{0}^{+}\left(\mathbf{x}_{0}, \mathbf{x}_{i}^{\prime}, t\right)$ is added to $f_{1,0}^{-}\left(\mathbf{x}_{0}^{\prime \prime}\right.$, $\left.\mathbf{x}_{i}^{\prime}, t\right)$, thus giving $f_{1,1}^{-}\left(\mathbf{x}_{0}^{\prime \prime}, \mathbf{x}_{i}^{\prime}, t\right)$. This is represented by the blue rays in Figure $9 \mathrm{c}$, which correspond to the main events in Figure 6d.

The interpretation of higher order iterations goes along similar lines. The ray diagram of the converged solution is shown in Figure 4a, in which the red and blue rays correspond to the main events in Figure 6e and 6f, respectively. Note a peculiar property of the ray diagram in Figure 4a: Instead of the upgoing blue rays giving rise to downward reflected rays at the interfaces, the downgoing red rays are launched from the surface, which meet the upgoing blue rays at the interfaces, in such a way that the downward reflected rays below the interfaces are annihilated. As a result, no rays other than the primary downgoing red rays reach the depth level $\partial \mathbb{D}_{i}$. This is in agreement with the definition of the focusing function, which should focus only at $\mathbf{x}_{i}^{\prime}$ on $\partial \mathbb{D}_{i}$ at $t=0$ (equation 6).

Note that, although for the interpretation of $f_{1, k}^{ \pm}\left(\mathbf{x}_{0}^{\prime \prime}, \mathbf{x}_{i}^{\prime}, t\right)$ we used the subsurface model to explain the different events, we did not use this model to obtain $f_{1, k}^{ \pm}\left(\mathbf{x}_{0}^{\prime \prime}, \mathbf{x}_{i}^{\prime}, t\right)$ in Figure 6. The scheme discussed in the previous sections uses only the reflection response $R^{\cup}\left(\mathbf{x}_{0}^{\prime \prime}, \mathbf{x}_{0}, t\right)$ (Figure 5c) and an estimate of the direct arrival of the Green's function, $G_{\mathrm{d}}\left(\mathbf{x}_{i}^{\prime}, \mathbf{x}_{0}^{\prime \prime}, t\right)$ (Figure 5d). All events appearing in $f_{1, k}^{ \pm}\left(\mathbf{x}_{0}^{\prime \prime}, \mathbf{x}_{i}^{\prime}, t\right)$, except the time-reversed direct arrival, are retrieved entirely from the reflection response $R^{\cup}\left(\mathbf{x}_{0}^{\prime \prime}, \mathbf{x}_{0}, t\right)$.
Next, we interpret equations 11 and 12, which are rewritten as

$$
\begin{aligned}
& \overbrace{G^{p,+}\left(\mathbf{x}_{0}^{\prime \prime}, \mathbf{x}_{i}^{\prime}, t\right)}^{t>t_{\mathrm{d}}\left(\mathbf{x}_{i}^{\prime}, \mathbf{x}_{0}^{\prime \prime}\right)}+\overbrace{f_{1}^{-}\left(\mathbf{x}_{0}^{\prime \prime}, \mathbf{x}_{i}^{\prime}, t\right)}^{t<t_{\mathrm{d}}\left(\mathbf{x}_{i}^{\prime}, \mathbf{x}_{0}^{\prime \prime}\right)} \\
& =\int_{\partial \mathbb{D}_{0}} \mathrm{~d} \mathbf{x}_{0} \int_{-\infty}^{t} R^{\cup}\left(\mathbf{x}_{0}^{\prime \prime}, \mathbf{x}_{0}, t-t^{\prime}\right) f_{1}^{+}\left(\mathbf{x}_{0}, \mathbf{x}_{i}^{\prime}, t^{\prime}\right) \mathrm{d} t^{\prime}
\end{aligned}
$$

and

$$
\begin{aligned}
& \overbrace{G^{p,-}\left(\mathbf{x}_{0}^{\prime \prime}, \mathbf{x}_{i}^{\prime}, t\right)}^{t \geq t_{\mathrm{d}}\left(\mathbf{x}_{i}^{\prime}, \mathbf{x}_{0}^{\prime \prime}\right)}-\overbrace{f_{1}^{+}\left(\mathbf{x}_{0}^{\prime \prime}, \mathbf{x}_{i}^{\prime},-t\right)}^{t \leq t_{\mathrm{d}}\left(\mathbf{x}_{i}^{\prime}, \mathbf{x}_{0}^{\prime \prime}\right)} \\
& =-\int_{\partial \mathbb{D}_{0}} \mathrm{~d} \mathbf{x}_{0} \int_{-\infty}^{t} R^{\cup}\left(\mathbf{x}_{0}^{\prime \prime}, \mathbf{x}_{0}, t-t^{\prime}\right) f_{1}^{-}\left(\mathbf{x}_{0}, \mathbf{x}_{i}^{\prime},-t^{\prime}\right) \mathrm{d} t^{\prime},
\end{aligned}
$$
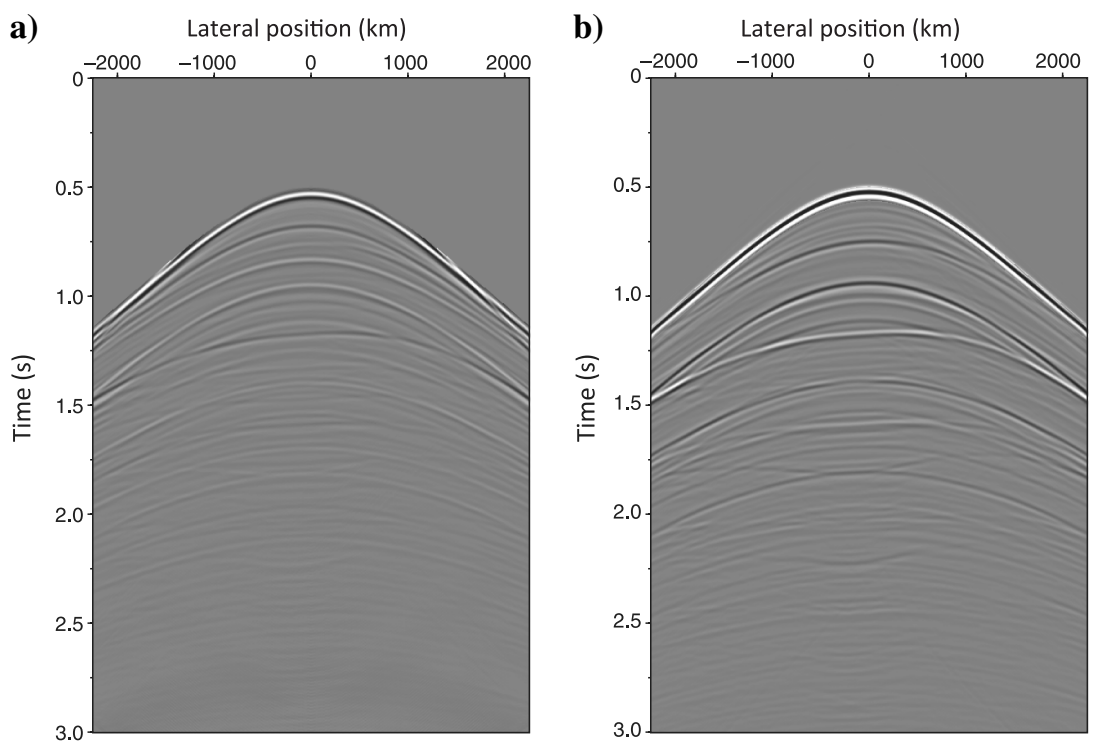

Figure 7. (a) The retrieved upgoing part of the Green's function, $G^{-}\left(\mathbf{x}_{i}^{\prime}, \mathbf{x}_{0}^{\prime \prime}, t\right)$, for a fixed virtual-receiver position $\mathbf{x}_{i}^{\prime}=(0,1100 \mathrm{~m})$ and variable source position $\mathbf{x}_{0}^{\prime \prime}$. (b) The retrieved downgoing part of the Green's function, $G^{+}\left(\mathbf{x}_{i}^{\prime}, \mathbf{x}_{0}^{\prime \prime}, t\right)$.

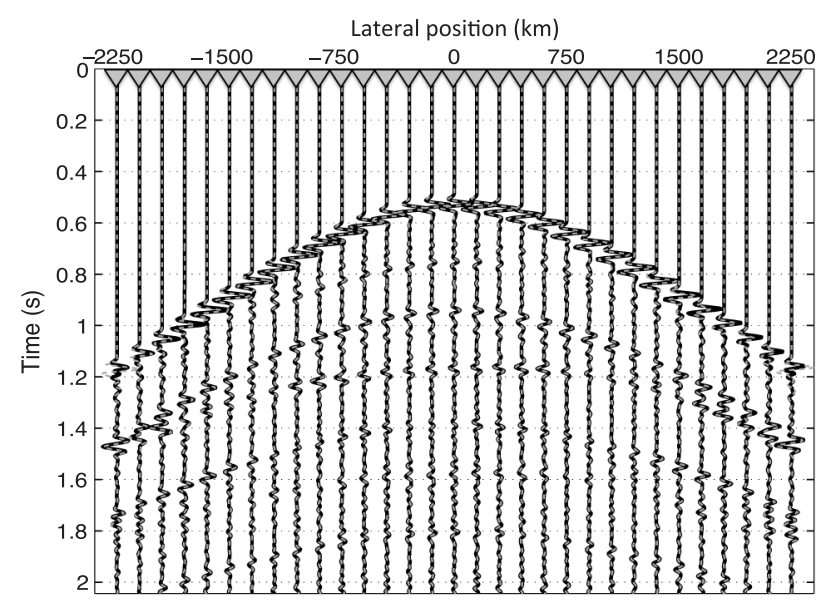

Figure 8. Green's functions, plotted in overlay. Black solid: Sum of the retrieved Green's functions $G^{-}\left(\mathbf{x}_{i}^{\prime}, \mathbf{x}_{0}^{\prime \prime}, t\right)$ and $G^{+}\left(\mathbf{x}_{i}^{\prime}, \mathbf{x}_{0}^{\prime \prime}, t\right)$ of Figure 7. Gray dashed: Directly modeled Green's function. Note that the black and gray dashed traces match each other almost perfectly, except for the far offsets of the first event. 
respectively. Note that we applied source-receiver reciprocity to the decomposed Green's functions on the left sides. We temporarily use two superscripts at the Green's functions. The first superscript $p$ refers to the observed quantity (acoustic pressure) at the observation point $\mathbf{x}_{0}^{\prime \prime}$; the second superscript (+ or - ) refers to the propagation direction at the virtual-source point $\mathbf{x}_{i}^{\prime}$. Figure $10 \mathrm{a}$ and $10 \mathrm{~b}$ represents the total responses on the left sides of equations 34 and 35 , respectively. The traveltime curve $t=t_{\mathrm{d}}\left(\mathbf{x}_{i}^{\prime}, \mathbf{x}_{0}^{\prime \prime}\right)$ is represented by the dashed lines in these figures.

The right side of equation 34 describes the reflection response of the actual medium to $f_{1}^{+}\left(\mathbf{x}_{0}, \mathbf{x}_{i}^{\prime}, t\right)$, for an unbounded time interval.
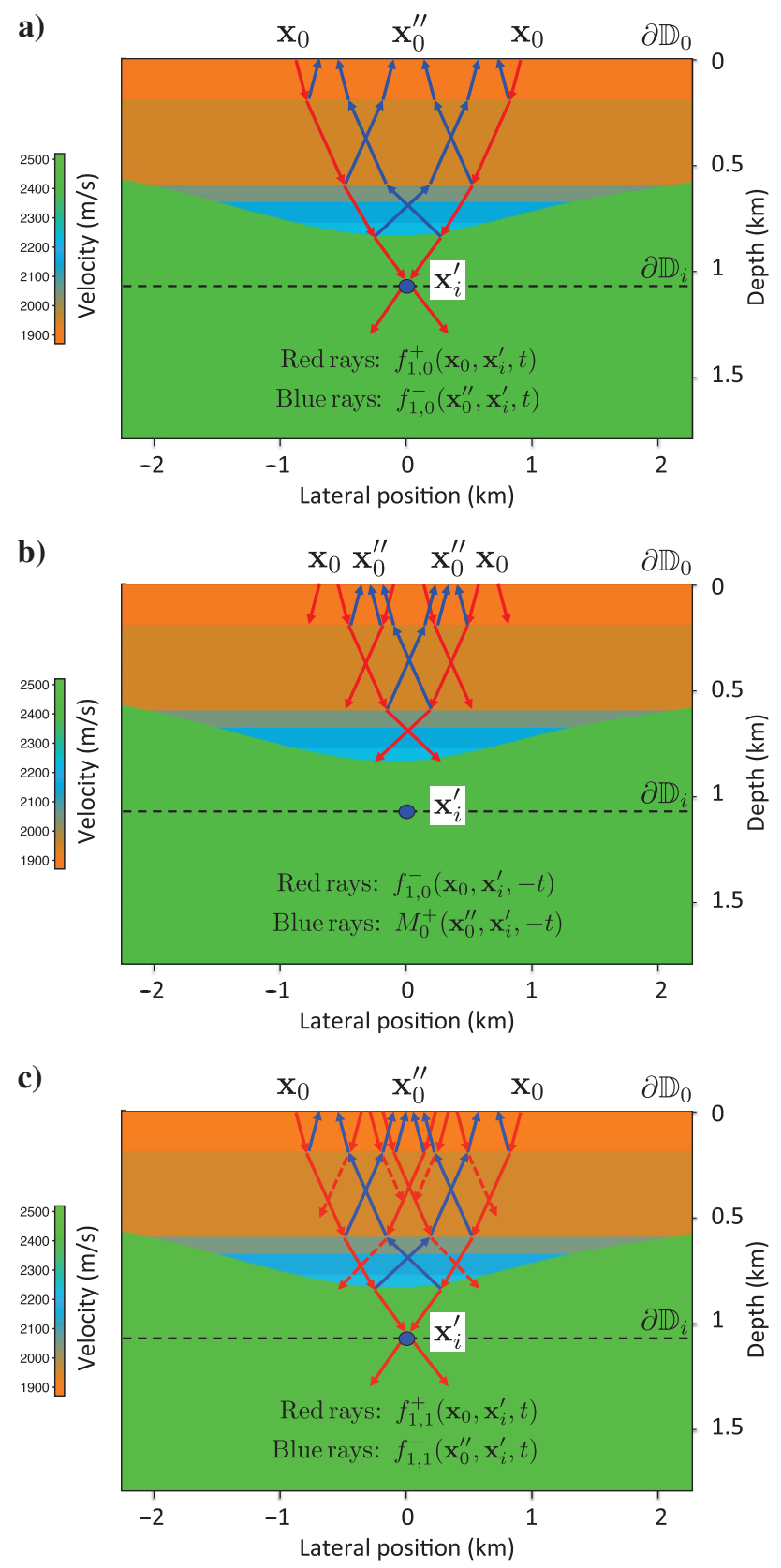

Figure 9. Physical interpretation of $f_{1, k}^{+}\left(\mathbf{x}_{0}, \mathbf{x}_{i}^{\prime}, t\right)$ and $f_{1, k}^{-}\left(\mathbf{x}_{0}^{\prime \prime}, \mathbf{x}_{i}^{\prime}\right.$, $t$ ), for $k=0$ and $k=1$. The ray diagrams in (a) and (c) explain the events in the focusing functions in Figure 6a-6d.
The downgoing red rays in Figure 11a represent the incident field $f_{1}^{+}\left(\mathbf{x}_{0}, \mathbf{x}_{i}^{\prime}, t\right)$, which focuses at $\mathbf{x}_{i}^{\prime}$, similar as in Figure 4a. The upgoing blue rays represent again the response $f_{1}^{-}\left(\mathbf{x}_{0}^{\prime \prime}, \mathbf{x}_{i}^{\prime}, t\right)$, with $t<t_{\mathrm{d}}\left(\mathbf{x}_{i}^{\prime}, \mathbf{x}_{0}^{\prime \prime}\right)$. Beyond the focal point, the downgoing rays diverge and after reflection arrive at the surface. This is illustrated by the green rays in Figure 11a, which represent the Green's function $G^{p,+}\left(\mathbf{x}_{0}^{\prime \prime}, \mathbf{x}_{i}^{\prime}, t\right), t>t_{\mathrm{d}}\left(\mathbf{x}_{i}^{\prime}, \mathbf{x}_{0}^{\prime \prime}\right)$, with its virtual source at the focal point $\mathbf{x}_{i}^{\prime}$. The left side of equation 34 represents the superposition of $f_{1}^{-}\left(\mathbf{x}_{0}^{\prime \prime}, \mathbf{x}_{i}^{\prime}, t\right)$ and $G^{p,+}\left(\mathbf{x}_{0}^{\prime \prime}, \mathbf{x}_{i}^{\prime}, t\right)$, see Figure 10a. By subtracting $f_{1}^{-}\left(\mathbf{x}_{0}^{\prime \prime}, \mathbf{x}_{i}^{\prime}, t\right)$, we obtain $G^{p,+}\left(\mathbf{x}_{0}^{\prime \prime}, \mathbf{x}_{i}^{\prime}, t\right)$ or after applying sourcereceiver reciprocity and returning to the single-superscript notation, $G^{-}\left(\mathbf{x}_{i}^{\prime}, \mathbf{x}_{0}^{\prime \prime}, t\right)$, see Figure 11b. This ray diagram represents the retrieved upgoing part of the Green's function in Figure 7a.

The interpretation of equation 35 is slightly more complicated. The right side describes the reflection response of the actual medium to $-f_{1}^{-}\left(\mathbf{x}_{0}, \mathbf{x}_{i}^{\prime},-t\right)$, which because of the time reversal, is a downgoing field. It is represented by the downgoing red rays in Figure 12a, which are the reversals of the upgoing blue rays in Figure 11a. The upgoing blue rays in Figure 12a show the response $-f_{1}^{+}\left(\mathbf{x}_{0}^{\prime \prime}, \mathbf{x}_{i}^{\prime},-t\right)$, for $t \leq t_{\mathrm{d}}\left(\mathbf{x}_{i}^{\prime}, \mathbf{x}_{0}^{\prime \prime}\right)$. The green-blue dashed ray represents the final arrival of $-f_{1}^{+}\left(\mathbf{x}_{0}^{\prime \prime}, \mathbf{x}_{i}^{\prime},-t\right)$ (i.e., the time-reversed first arrival of $\left.-f_{1}^{+}\left(\mathbf{x}_{0}^{\prime \prime}, \mathbf{x}_{i}^{\prime}, t\right)\right)$. Its path is shared with that of the direct arrival of the Green's function $G^{p,-}\left(\mathbf{x}_{0}^{\prime \prime}, \mathbf{x}_{i}^{\prime}, t\right)$. The downgoing red rays cross the paths of the direct wave and give rise to the multiple reflections of the Green's function $G^{p,-}\left(\mathbf{x}_{0}^{\prime \prime}\right.$, $\left.\mathbf{x}_{i}^{\prime}, t\right)$, represented by the solid green rays in Figure 12a. Note that all rays associated with $G^{p,-}\left(\mathbf{x}_{0}^{\prime \prime}, \mathbf{x}_{i}^{\prime}, t\right)$ apparently originate from a virtual source at the focal point $\mathbf{x}_{i}^{\prime}$. The left side of equation 35 represents the superposition of $-f_{1}^{+}\left(\mathbf{x}_{0}^{\prime \prime}, \mathbf{x}_{i}^{\prime},-t\right)$ and $G^{p,-}\left(\mathbf{x}_{0}^{\prime \prime}, \mathbf{x}_{i}^{\prime}, t\right)$, see Figure $10 \mathrm{~b}$. By subtracting $-f_{1}^{+}\left(\mathbf{x}_{0}^{\prime \prime}, \mathbf{x}_{i}^{\prime},-t\right)$, we obtain $G^{p,-}\left(\mathbf{x}_{0}^{\prime \prime}, \mathbf{x}_{i}^{\prime}, t\right)$ or after applying source-receiver reciprocity and returning to the single-superscript notation, $G^{+}\left(\mathbf{x}_{i}^{\prime}, \mathbf{x}_{0}^{\prime \prime}, t\right)$, see Figure $12 \mathrm{~b}$. This ray diagram represents the retrieved downgoing part of the Green's function in Figure $7 \mathrm{~b}$.

\section{MARCHENKO REDATUMING}

"Redatuming" refers to the process of virtually moving sources and receivers from a specific datum plane (typically the acquisition surface) to a new datum plane inside the medium, closer to a target zone. In classical redatuming (Berryhill, 1979, 1984; Kinneging et al., 1989), one-way primary operators are used to redatum the reflection response from $\partial \mathbb{D}_{0}$ to $\partial \mathbb{D}_{i}$. These operators are based on a background model of the medium between $\partial \mathbb{D}_{0}$ and $\partial \mathbb{D}_{i}$. These operators do not account for internal multiple reflections; hence, a classically redatumed response contains spurious reflections (i.e., reflections that do not belong to the reflection response at $\partial \mathbb{D}_{i}$ ).

Redatuming can be seen as a two-step process: starting with sources and receivers at the surface $\partial \mathbb{D}_{0}$; in the first step, the receivers are moved from $\partial \mathbb{D}_{0}$ to $\partial \mathbb{D}_{i}$, followed by moving the sources to $\partial \mathbb{D}_{i}$ in the second step. In some cases, acquisition is carried out with sources at the surface $\partial \mathbb{D}_{0}$ and receivers at a datum plane $\partial \mathbb{D}_{i}$ inside the medium. For example, receivers may be located in a horizontal borehole. For this situation, redatuming is a one-step process, bringing only the sources from $\partial \mathbb{D}_{0}$ to $\partial \mathbb{D}_{i}$ (Bakulin and Calvert, 2006). No information about the medium is needed because the receivers at $\partial \mathbb{D}_{i}$ measure the required downward extrapolation operators. This data-driven approach to redatuming is called interferometric redatuming (Schuster and Zhou, 2006). Because the operators for interferometric redatuming are measured, they account, in principle, for 
a)

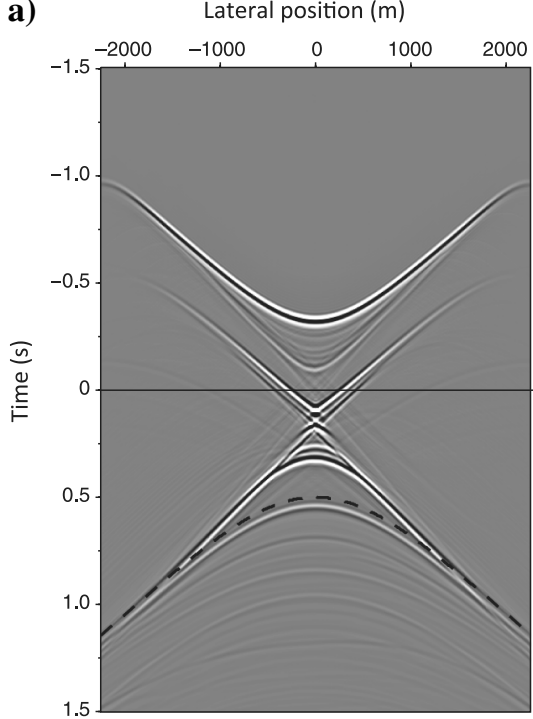

b)

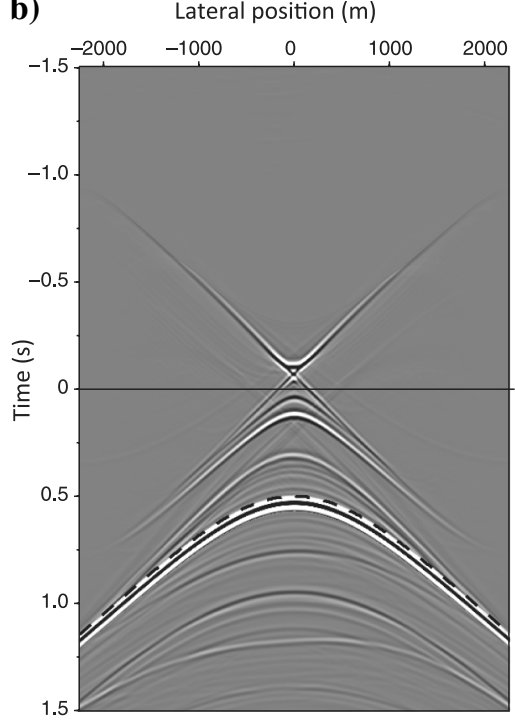

Figure 10. (a) The reflection response of the actual medium to $f_{1}^{+}\left(\mathbf{x}_{0}, \mathbf{x}_{i}^{\prime}, t\right)$ (equation 34). (b) The reflection response of the actual medium to $-f_{1}^{-}\left(\mathbf{x}_{0}, \mathbf{x}_{i}^{\prime},-t\right)$ (equation 35).

internal multiple reflections. This property is exploited by a method called interferometric redatuming by multidimensional deconvolution $(M D D)$, which indeed leads to a ghost-free reflection response at $\partial \mathbb{D}_{i}$ (van der Neut et al., 2011).

In most situations, however, sources and receivers are situated at the surface. The iterative Marchenko scheme discussed in the foregoing sections is applied to the reflection response $R^{\cup}\left(\mathbf{x}_{0}^{\prime \prime}, \mathbf{x}_{0}, t\right)$ at the surface $\partial \mathbb{D}_{0}$, yielding the Green's functions $G^{-}\left(\mathbf{x}_{i}^{\prime}, \mathbf{x}_{0}^{\prime \prime}, t\right)$ and $G^{+}\left(\mathbf{x}_{i}^{\prime}, \mathbf{x}_{0}^{\prime \prime}\right.$, $t)$, i.e., responses to sources at the surface $\partial \mathbb{D}_{0}$, observed by virtual receivers at datum plane $\partial \mathbb{D}_{i}$ inside the medium. This can be seen as the first step of a two-step redatuming process. This form of receiver redatuming requires an estimate of the direct arrival of the inverse transmission response, $\mathcal{T}_{\mathrm{d}}^{\text {inv }}\left(\mathbf{x}_{i}^{\prime}, \mathbf{x}_{0}^{\prime \prime}, t\right)$. This is actually the same as the one-way primary operator used in classical redatuming. The direct arrivals can, for example, be obtained by modeling in a background model
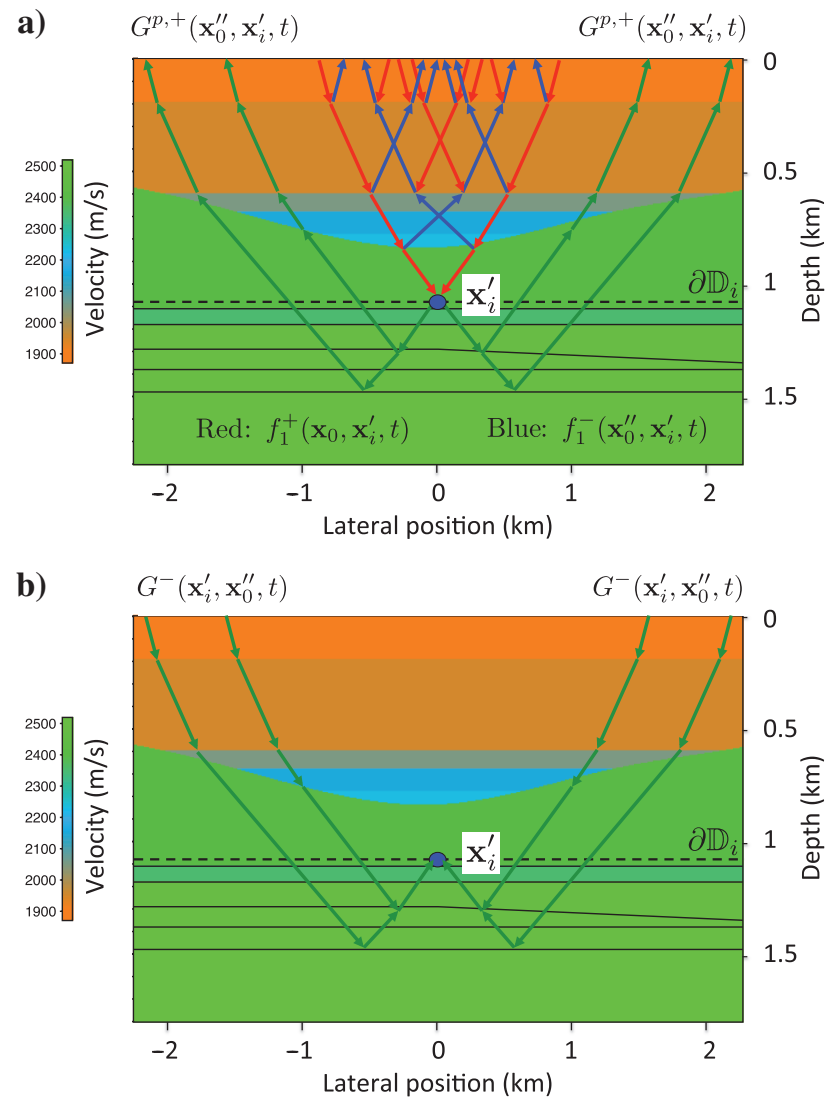

Figure 11. Physical interpretation of equation 34. (a) The incident field $f_{1}^{+}\left(\mathbf{x}_{0}, \mathbf{x}_{i}^{\prime}, t\right)$ focuses at $\mathbf{x}_{i}^{\prime}$ (red rays). Its response consists of $f_{1}^{-}\left(\mathbf{x}_{0}^{\prime \prime}, \mathbf{x}_{i}^{\prime}, t\right)$ (blue rays) and the Green's function $G^{p,+}\left(\mathbf{x}_{0}^{\prime \prime}, \mathbf{x}_{i}^{\prime}, t\right)$ (green rays). (b) After subtracting $f_{1}^{-}\left(\mathbf{x}_{0}^{\prime \prime}, \mathbf{x}_{i}^{\prime}, t\right)$ and applying sourcereceiver reciprocity, the Green's function $G^{-}\left(\mathbf{x}_{i}^{\prime}, \mathbf{x}_{0}^{\prime \prime}, t\right)$ remains.
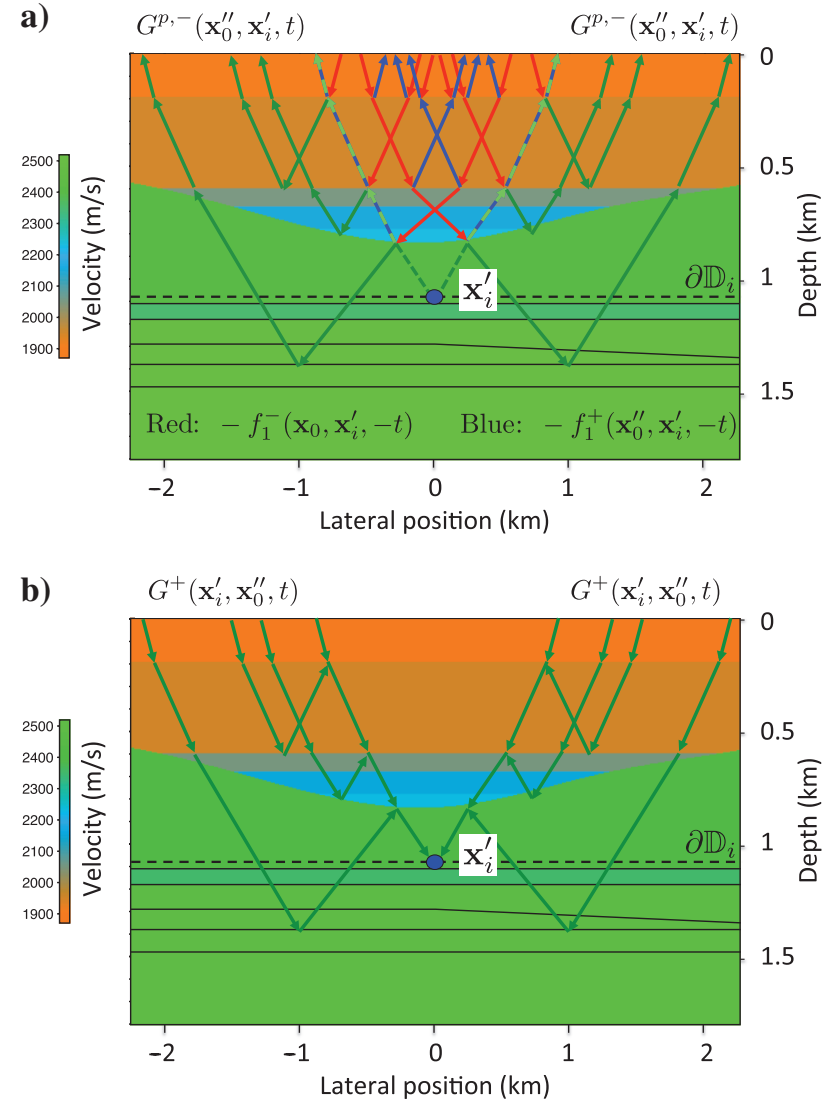

Figure 12. Physical interpretation of equation 35. (a) The incident field $-f_{1}^{-}\left(\mathbf{x}_{0}, \mathbf{x}_{i}^{\prime},-t\right)$ (red rays) gives rise to $-f_{1}^{+}\left(\mathbf{x}_{0}^{\prime \prime}, \mathbf{x}_{i}^{\prime},-t\right)$ (blue rays) and the Green's function $G^{p,-}\left(\mathbf{x}_{0}^{\prime \prime}, \mathbf{x}_{i}^{\prime}, t\right)$ (green rays). (b) After subtracting $-f_{1}^{+}\left(\mathbf{x}_{0}^{\prime \prime}, \mathbf{x}_{i}^{\prime},-t\right)$ and applying source-receiver reciprocity, the Green's function $G^{+}\left(\mathbf{x}_{i}^{\prime}, \mathbf{x}_{0}^{\prime \prime}, t\right)$ remains. 
between $\partial \mathbb{D}_{0}$ and $\partial \mathbb{D}_{i}$ or, when $\partial \mathbb{D}_{i}$ coincides with an actual reflector, they can be derived from the reflection response at the surface via the common-focal-point method (Berkhout, 1997; Berkhout and Verschuur, 2001). Note that, unlike the first step in classical redatuming, our iterative form of receiver redatuming accounts for internal multiple reflections. Once the receivers have been redatumed to $\partial \mathbb{D}_{i}$, the sources can be redatumed to $\partial \mathbb{D}_{i}$ in a similar way as in interferometric redatuming by MDD. We call the combination of these two steps Marchenko redatuming. In the remainder of this section, the source redatuming step is discussed in more detail.

The Green's functions $G^{-}\left(\mathbf{x}_{i}^{\prime}, \mathbf{x}_{0}^{\prime \prime}, t\right)$ and $G^{+}\left(\mathbf{x}_{i}^{\prime}, \mathbf{x}_{0}^{\prime \prime}, t\right)$ are mutually related via the reflection response of the medium below $\partial \mathbb{D}_{i}$, according to

$G^{-}\left(\mathbf{x}_{i}, \mathbf{x}_{0}^{\prime \prime}, t\right)=\int_{\partial \mathbb{D}_{i}} \mathrm{~d} \mathbf{x}_{i}^{\prime} \int_{-\infty}^{\infty} R^{\cup}\left(\mathbf{x}_{i}, \mathbf{x}_{i}^{\prime}, t^{\prime}\right) G^{+}\left(\mathbf{x}_{i}^{\prime}, \mathbf{x}_{0}^{\prime \prime}, t-t^{\prime}\right) \mathrm{d} t^{\prime}$

(Wapenaar et al., 2000; Amundsen, 2001). This expression states that the downgoing field $G^{+}$at $\partial \mathbb{D}_{i}$, convolved with the reflection response $R^{\cup}$ at $\partial \mathbb{D}_{i}$ and integrated along all source positions of this reflection response at $\partial \mathbb{D}_{i}$, gives the upgoing field $G^{-}$at $\partial \mathbb{D}_{i}$. Note that the fields $G^{-}$and $G^{+}$are defined in the actual medium (Figure 1), whereas the reflection response $R^{\cup}$ is defined in a reference medium that is identical to the actual medium below $\partial \mathbb{D}_{i}$ and reflection-free above this depth level.

For each $\mathbf{x}_{0}^{\prime \prime}$, equation 36 is an integral equation for the reflection response $R^{\cup}\left(\mathbf{x}_{i}, \mathbf{x}_{i}^{\prime}, t\right)$. Figure 13a shows ray diagrams of $G^{-}\left(\mathbf{x}_{i}, \mathbf{x}_{0}^{\prime \prime}, t\right)$ and $G^{+}\left(\mathbf{x}_{i}^{\prime}, \mathbf{x}_{0}^{\prime \prime}, t\right)$ for fixed $\mathbf{x}_{0}^{\prime \prime}$ and variable $\mathbf{x}_{i}$ and $\mathbf{x}_{i}^{\prime} . R^{\cup}\left(\mathbf{x}_{i}, \mathbf{x}_{i}^{\prime}, t\right)$ can be resolved from equation 36 by MDD when the Green's functions are available for many source positions $\mathbf{x}_{0}^{\prime \prime}$. To this end, both sides of equation 36 are first correlated with the downgoing Green's function and integrated over $\mathbf{x}_{0}^{\prime \prime} \in \partial \mathbb{D}_{0}$. This yields

$$
C\left(\mathbf{x}_{i}, \mathbf{x}_{i}^{\prime \prime \prime}, t\right)=\int_{\partial \mathbb{D}_{i}} \mathrm{~d} \mathbf{x}_{i}^{\prime} \int_{-\infty}^{\infty} R^{\cup}\left(\mathbf{x}_{i}, \mathbf{x}_{i}^{\prime}, t^{\prime}\right) \Gamma\left(\mathbf{x}_{i}^{\prime}, \mathbf{x}_{i}^{\prime \prime \prime}, t-t^{\prime}\right) \mathrm{d} t^{\prime},
$$

with the correlation function $C\left(\mathbf{x}_{i}, \mathbf{x}_{i}^{\prime \prime \prime}, t\right)$ and point-spread function $\Gamma\left(\mathbf{x}_{i}^{\prime}, \mathbf{x}_{i}^{\prime \prime \prime}, t\right)$ defined as

$$
\begin{aligned}
& C\left(\mathbf{x}_{i}, \mathbf{x}_{i}^{\prime \prime \prime}, t\right)= \\
& \quad \int_{\partial \mathbb{D}_{0}} \mathrm{~d} \mathbf{x}_{0}^{\prime \prime} \int_{-\infty}^{\infty} G^{-}\left(\mathbf{x}_{i}, \mathbf{x}_{0}^{\prime \prime}, t+t^{\prime}\right) G^{+}\left(\mathbf{x}_{i}^{\prime \prime \prime}, \mathbf{x}_{0}^{\prime \prime}, t^{\prime}\right) \mathrm{d} t^{\prime}
\end{aligned}
$$

and

$$
\begin{aligned}
& \Gamma\left(\mathbf{x}_{i}^{\prime}, \mathbf{x}_{i}^{\prime \prime \prime}, t\right)= \\
& \quad \int_{\partial \mathbb{D}_{0}} \mathrm{~d} \mathbf{x}_{0}^{\prime \prime} \int_{-\infty}^{\infty} G^{+}\left(\mathbf{x}_{i}^{\prime}, \mathbf{x}_{0}^{\prime \prime}, t+t^{\prime}\right) G^{+}\left(\mathbf{x}_{i}^{\prime \prime \prime}, \mathbf{x}_{0}^{\prime \prime}, t^{\prime}\right) \mathrm{d} t^{\prime},
\end{aligned}
$$

respectively. MDD involves inverting equation 37 . This gives the redatumed reflection response $R^{\cup}\left(\mathbf{x}_{i}, \mathbf{x}_{i}^{\prime}, t\right)$, with virtual sources $\left(\mathbf{x}_{i}^{\prime}\right)$ and virtual receivers $\left(\mathbf{x}_{i}\right)$ at $\partial \mathbb{D}_{i}$. For the practical aspects of this inversion, see van der Neut et al. (2011). As mentioned before, this deconvolution process largely compensates for the amplitude errors in $G^{-}\left(\mathbf{x}_{i}, \mathbf{x}_{0}^{\prime \prime}, t\right)$ and $G^{+}\left(\mathbf{x}_{i}^{\prime}, \mathbf{x}_{0}^{\prime \prime}, t\right)$, related to approximating the direct arrival of the inverse transmission response by the time reversal of the direct arrival of the Green's function (equation 18).

We illustrate this source redatuming procedure by applying MDD to the retrieved Green's functions $G^{-}\left(\mathbf{x}_{i}, \mathbf{x}_{0}^{\prime \prime}, t\right)$ and $G^{+}\left(\mathbf{x}_{i}^{\prime}, \mathbf{x}_{0}^{\prime \prime}, t\right)$ of Figure 7 . To this end, we repeat the receiver redatuming procedure that led to Figure 7 for a range of $\mathbf{x}_{i}^{\prime}$-values along $\partial \mathbb{D}_{i}$. Next, we evaluate the correlation and point-spread functions of equations 38 and 39, respectively, and invert equation 37. The result, $R^{\cup}\left(\mathbf{x}_{i}\right.$, $\left.\mathbf{x}_{i}^{\prime}, t\right)$, is shown in Figure 13b, for fixed $\mathbf{x}_{i}^{\prime}=(0,1100 \mathrm{~m})$ and variable $\mathbf{x}_{i}=\left(x_{1}, x_{3, i}=1100 \mathrm{~m}\right)$, with $x_{1}$ ranging from $-2000 \mathrm{~m}$ to a)

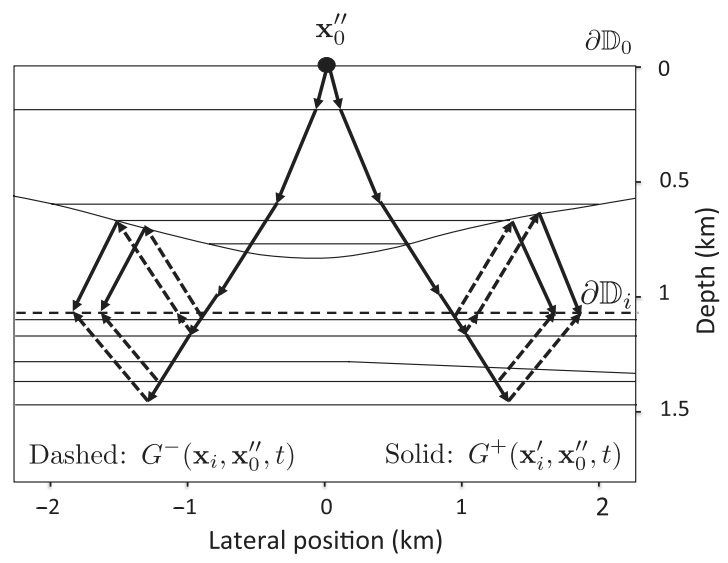

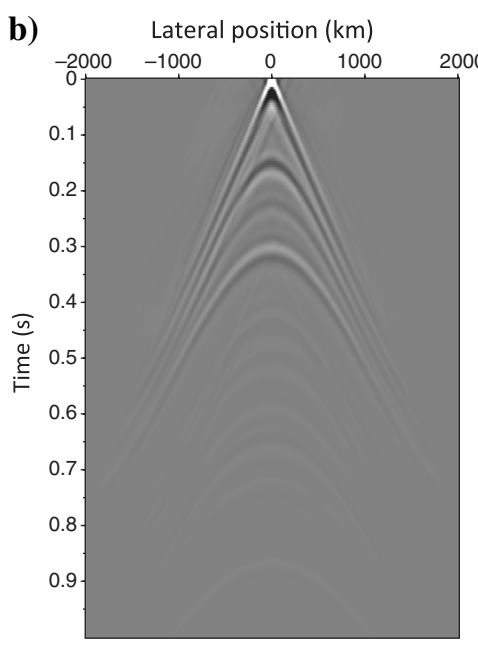

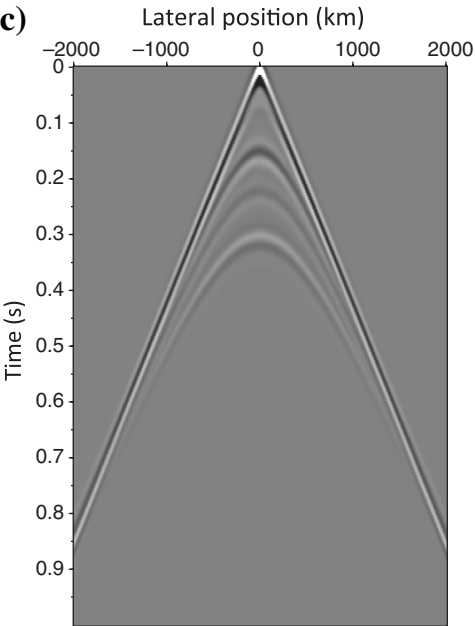

Figure 13. (a) Ray diagram of the Green's functions $G^{-}\left(\mathbf{x}_{i}, \mathbf{x}_{0}^{\prime \prime}, t\right)$ and $G^{+}\left(\mathbf{x}_{i}^{\prime}, \mathbf{x}_{0}^{\prime \prime}, t\right)$. These fields are used to retrieve the reflection response $R^{\cup}\left(\mathbf{x}_{i}, \mathbf{x}_{i}^{\prime}, t\right)$ of the target area below $\partial \mathbb{D}_{i}$. (b) Marchenko redatuming result $R^{\cup}\left(\mathbf{x}_{i}, \mathbf{x}_{i}^{\prime}, t\right)$, with $\mathbf{x}_{i}^{\prime}=(0,1100 \mathrm{~m})$ at the top of the target zone. (c) Directly modeled shot record, for a source at $\mathbf{x}_{i}^{\prime}$. 
$2000 \mathrm{~m}$ (i.e., the top of the target zone). Figure $13 \mathrm{c}$ shows a directly modeled shot record, with its source at $\mathbf{x}_{i}^{\prime}$. Note that the retrieved response (Figure 13b) clearly exhibits the reflections of the layers in the target zone, with hardly any contamination by internal multiples of the overburden.

Summarizing, we have presented Marchenko redatuming of the reflection response from the surface $\partial \mathbb{D}_{0}$ to $\partial \mathbb{D}_{i}$ inside the medium, as a two-step process. Step one, using the iterative Marchenko scheme, moves the receivers from $\partial \mathbb{D}_{0}$ to $\partial \mathbb{D}_{i}$ (as illustrated with the numerical example in Figure $7 \mathrm{a}$ and $7 \mathrm{~b}$ ). Step two, interferometric redatuming by $\mathrm{MDD}$, moves the sources from $\partial \mathbb{D}_{0}$ to $\partial \mathbb{D}_{i}$ (as illustrated in Figure 13b). This two-step redatuming process requires the same input as classical redatuming (i.e., the reflection response at the surface $\partial \mathbb{D}_{0}$ and an estimate of the direct arrivals between $\partial \mathbb{D}_{0}$ and $\partial \mathbb{D}_{i}$ ), but, unlike classical redatuming, it uses the internal multiples in the original reflection response $R^{\cup}\left(\mathbf{x}_{0}^{\prime \prime}\right.$, $\left.\mathbf{x}_{0}, t\right)$ to obtain the redatumed reflection response $R^{\cup}\left(\mathbf{x}_{i}, \mathbf{x}_{i}^{\prime}, t\right)$ at $\partial \mathbb{D}_{i}$, free of spurious reflections related to internal multiples in the overburden.

Until now, it was assumed that $R^{\cup}\left(\mathbf{x}_{0}^{\prime \prime}, \mathbf{x}_{0}, t\right)$ is measured in a configuration with a homogeneous upper half-space above $\partial \mathbb{D}_{0}$ (Figure 1). In many cases, the acquisition surface $\partial \mathbb{D}_{0}$ is a free surface, meaning that $R^{\cup}\left(\mathbf{x}_{0}^{\prime \prime}, \mathbf{x}_{0}, t\right)$ is contaminated with surfacerelated multiples. In those cases, Marchenko redatuming should be preceded by surface-related multiple elimination (SRME) (Verschuur et al., 1992; van Groenestijn and Verschuur, 2009). The output of SRME is the reflection response $R^{\cup}\left(\mathbf{x}_{0}^{\prime \prime}, \mathbf{x}_{0}, t\right)$ in the configuration of Figure 1.

\section{MARCHENKO IMAGING}

\section{Imaging from above}

The redatumed reflection response $R^{\cup}\left(\mathbf{x}_{i}, \mathbf{x}_{i}^{\prime}, t\right)$ can be used in different ways for reflection imaging. The most straightforward approach is to extract the zero-offset $\left(\mathbf{x}_{i}^{\prime}=\mathbf{x}_{i}\right)$, zero-time ( $\left.t=0\right)$ component, i.e., $R^{\cup}\left(\mathbf{x}_{i}, \mathbf{x}_{i}, 0\right)$, repeat the process for all $\mathbf{x}_{i}$ at a wellsampled range of depth levels $x_{3, i}$ in the region of interest, and thus construct an image of the zero-offset reflectivity $R^{\cup}(\mathbf{x}, \mathbf{x}, 0)$. We call this Marchenko imaging. Because $R^{\cup}\left(\mathbf{x}_{i}, \mathbf{x}_{i}^{\prime}, t\right)$ is the correctly redatumed reflection response, the image thus obtained will be free of spurious reflections (Wapenaar et al., 2012). Note that this method can start and end at any two depth levels, leading to a ghost-free image of the target zone between these depth levels, without the need to derive a detailed model of the medium between the acquisition surface and the target zone; as in standard imaging, a smooth subsurface model that explains the direct arrivals suffices.

Instead of selecting only the zero-offset, zero-time component $R^{\cup}\left(\mathbf{x}_{i}, \mathbf{x}_{i}, 0\right)$, the full reflection response $R^{\cup}\left(\mathbf{x}_{i}, \mathbf{x}_{i}^{\prime}, t\right)$ can be used to derive the angle-dependent reflectivity at each image point (de Bruin et al., 1990; Sava and Fomel, 2003). This information can be used as input for amplitude-versus-angle inversion to determine the detailed medium parameters in the target zone.

Applying Marchenko redatuming to many depth levels in a target zone is a costly process. Alternatively, a relatively small number of sparse depth levels can be selected to which the reflection response is redatumed. Subsequently, standard imaging can be applied to the redatumed reflection responses $R^{\cup}\left(\mathbf{x}_{i}, \mathbf{x}_{i}^{\prime}, t\right)$ to image the regions between the selected depth levels. Assuming the depth levels are selected with care (for example, between the major reflecting boun- daries), this will still lead to a nearly ghost-free image. This approach can be used to image below a complicated near-surface, or for subsalt imaging.

Resolving $R^{\cup}\left(\mathbf{x}_{i}, \mathbf{x}_{i}^{\prime}, t\right)$ from equation 37 is an inversion process, which may sometimes be unstable. As an alternative, consider the correlation function $C\left(\mathbf{x}_{i}, \mathbf{x}_{i}^{\prime}, t\right)$, defined in equation 38 . The correlation integral on the right side resembles the definition of interferometric redatuming by crosscorrelation of decomposed data (Mehta et al., 2007), except that equation 38 contains the Green's functions obtained by Marchenko redatuming. The first event in the correlation function $C\left(\mathbf{x}_{i}, \mathbf{x}_{i}^{\prime}, t\right)$ is a first-order approximation of the first event of the reflection response $R^{\cup}\left(\mathbf{x}_{i}, \mathbf{x}_{i}^{\prime}, t\right)$. An image constructed from $C(\mathbf{x}, \mathbf{x}, 0)$ in a specific target zone will not have the correct amplitudes but will still be free of spurious reflections (Wapenaar et al., 2012). Moreover, the multiples in $G^{-}\left(\mathbf{x}_{i}, \mathbf{x}_{0}^{\prime \prime}, t\right)$ and $G^{+}\left(\mathbf{x}_{i}^{\prime}, \mathbf{x}_{0}^{\prime \prime}, t\right)$ contribute to the image (Behura et al., 2012). Alternatively, for a carefully selected range of sparse depth levels, standard imaging can be applied to $C\left(\mathbf{x}_{i}, \mathbf{x}_{i}^{\prime}, t\right)$ to image the regions between the selected depth levels. Because $C\left(\mathbf{x}_{i}, \mathbf{x}_{i}^{\prime}, t\right)$ seldom has the correct amplitude information, it cannot be used for angle-dependent reflection imaging.

Broggini et al. (2014) show an example of a comparison between different imaging approaches and investigate the sensitivity of Marchenko imaging with respect to errors in the estimate of the direct arrivals. Here, we illustrate target-oriented imaging with a numerical example. In Figure 13b, we showed the redatumed response $R^{\cup}\left(\mathbf{x}_{i}, \mathbf{x}_{i}^{\prime}, t\right)$ at the top of the target zone, for $\mathbf{x}_{i}^{\prime}=\left(x_{1}^{\prime}=\right.$ $\left.0, x_{3, i}=1100 \mathrm{~m}\right)$. Similar responses are computed for a range of $x_{1}^{\prime}$ values, between -2000 and $2000 \mathrm{~m}$. We use these redatumed responses as input for standard imaging in the target zone. The result is shown in Figure 14a, which clearly shows the reflectors of the target zone. For comparison, Figure 14b shows the same target zone, selected from a standard image obtained from the reflection response at the surface, $R^{\cup}\left(\mathbf{x}_{0}^{\prime \prime}, \mathbf{x}_{0}, t\right)$ (in both figures, amplitudes greater than $25 \%$ of the maximum amplitude have been clipped; no depth-dependent gain has been applied). Whereas the latter result is contaminated by spurious reflections, caused by internal multiples in the overburden, our image result in Figure 14a is free of these contaminations. Note that processes like full-waveform inversion (Virieux and Operto, 2009) and full wavefield migration (Berkhout and Verschuur, 2011) might lead to results similar as the one shown in Figure 14a. A detailed comparison with these methods is beyond the scope of this paper. We only mention one important difference. Methods like full-waveform inversion/migration are recursive methods, meaning that deep layers can only be imaged after the shallower structures have been resolved. In contrast, Marchenko imaging is a nonrecursive method: the image in Figure 14a has been obtained without using information about the overburden, other than the smooth model of Figure 5b.

Several other approaches exist that deal with internal multiples (Weglein et al., 1997, 2011; Berkhout and Verschuur, 1997, 2011; Jakubowicz, 1998; Ten Kroode, 2002; Brookes, 2011). The relation between Marchenko imaging and these methods needs further investigation. As in standard imaging, the exclusion of evanescent waves in the focusing functions implies a limitation to the maximum obtainable spatial resolution (Berkhout and van Wulfften Palthe, 1979). In principle, this resolution limit can be overcome by taking evanescent waves into account (Williams and Maynard, 1980; Lerosey et al., 2007; Fink and Tanter, 2010; Cao et al., 
2012). It remains to be investigated to what extent evanescent waves can be used to improve the resolution of Marchenko imaging.

\section{Imaging from below}

The decomposed Marchenko scheme not only derives the oneway Green's functions from the reflection response at the surface, it also yields the one-way focusing functions $f_{1}^{+}\left(\mathbf{x}_{0}^{\prime \prime}, \mathbf{x}_{j}^{\prime}, t\right)$ and $f_{1}^{-}\left(\mathbf{x}_{0}^{\prime \prime}, \mathbf{x}_{j}, t\right)$ and, via equations 9 and 10 , the focusing functions $f_{2}^{-}\left(\mathbf{x}_{j}^{\prime}, \mathbf{x}_{0}^{\prime \prime}, t\right)$ and $f_{2}^{+}\left(\mathbf{x}_{j}, \mathbf{x}_{0}^{\prime \prime}, t\right)$. Whereas the one-way Green's functions are mutually related via the reflection response to downgoing waves of the medium below $\partial \mathbb{D}_{i}$ (see equation 36), the one-way focusing functions $f_{2}^{-}\left(\mathbf{x}_{j}^{\prime}, \mathbf{x}_{0}^{\prime \prime}, t\right)$ and $f_{2}^{+}\left(\mathbf{x}_{j}, \mathbf{x}_{0}^{\prime \prime}, t\right)$ (Figure 2b) are related via the reflection response to upgoing waves of the medium above $\partial \mathbb{D}_{j}$, according to

$$
f_{2}^{+}\left(\mathbf{x}_{j}, \mathbf{x}_{0}^{\prime \prime}, t\right)=\int_{\partial \mathbb{D}_{j}} \mathrm{~d} \mathbf{x}_{j}^{\prime} \int_{-\infty}^{\infty} R^{\cap}\left(\mathbf{x}_{j}, \mathbf{x}_{j}^{\prime}, t^{\prime}\right) f_{2}^{-}\left(\mathbf{x}_{j}^{\prime}, \mathbf{x}_{0}^{\prime \prime}, t-t^{\prime}\right) \mathrm{d} t^{\prime}
$$

(see Appendix A, equation A-17). For each $\mathbf{x}_{0}^{\prime \prime}$, equation 40 is an integral equation for the reflection response $R^{\cap}\left(\mathbf{x}_{j}, \mathbf{x}_{j}^{\prime}, t\right)$. Figure 15 a
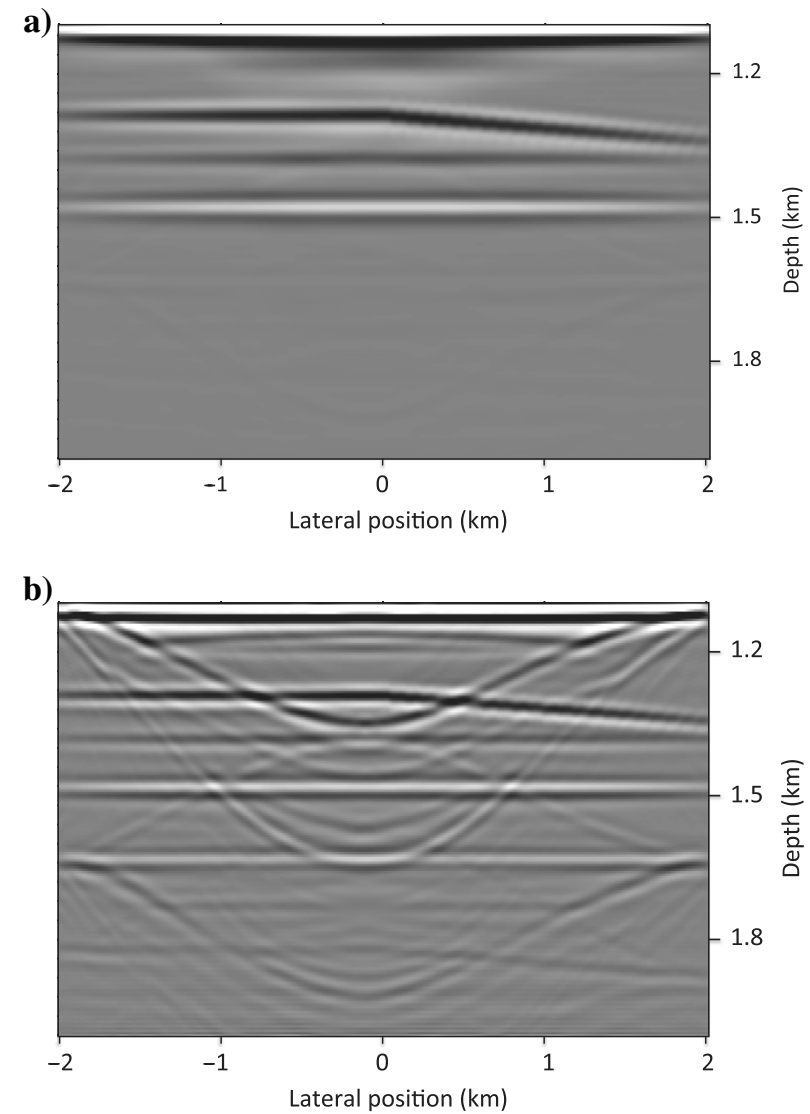

Figure 14. (a) Marchenko image of the target zone, obtained from the redatumed response $R^{\cup}\left(\mathbf{x}_{i}, \mathbf{x}_{i}^{\prime}, t\right)$. Note that this image has been obtained without first resolving the reflection properties of the overburden. (b) Standard image, obtained from the reflection response $R^{\cup}\left(\mathbf{x}_{0}^{\prime \prime}, \mathbf{x}_{0}, t\right)$ at the surface. shows ray diagrams of the focusing functions $f_{2}^{-}\left(\mathbf{x}_{j}^{\prime}, \mathbf{x}_{0}^{\prime \prime}, t\right)$ and $f_{2}^{+}\left(\mathbf{x}_{j}, \mathbf{x}_{0}^{\prime \prime}, t\right)$, for fixed $\mathbf{x}_{0}^{\prime \prime}$ and variable $\mathbf{x}_{j}$ and $\mathbf{x}_{j}^{\prime}$ at $\partial \mathbb{D}_{j}$, with $x_{3, j}=1600 \mathrm{~m}$. Figure $15 \mathrm{~b}$ and $15 \mathrm{c}$ shows numerical examples of these functions, obtained from the reflection response $R^{\cup}\left(\mathbf{x}_{0}^{\prime \prime}, \mathbf{x}_{0}, t\right)$ of Figure 5c by applying five iterations of the Marchenko scheme. The variable $\mathbf{x}_{j}^{\prime}$ is defined as $\mathbf{x}_{j}^{\prime}=\left(x_{1}^{\prime}\right.$, $\left.x_{3, j}=1600 \mathrm{~m}\right)$, with $x_{1}^{\prime}$ ranging from -2000 to $2000 \mathrm{~m}$. $R^{\cap}\left(\mathbf{x}_{j}, \mathbf{x}_{j}^{\prime}, t\right)$ can be resolved from equation 40 by MDD, when the focusing functions are available for many focal points $\mathbf{x}_{0}^{\prime \prime}$. The procedure is the same as for resolving $R^{\cup}\left(\mathbf{x}_{i}, \mathbf{x}_{i}^{\prime}, t\right)$ from equation 36. Once $R^{\cap}\left(\mathbf{x}_{j}, \mathbf{x}_{j}^{\prime}, t\right)$ has been obtained for all $\mathbf{x}_{j}^{\prime}$ at the bottom of the target zone, it can be used to image reflectors at and above $\partial \mathbb{D}_{j}$ from below, in a similar way as $R^{\cup}\left(\mathbf{x}_{i}, \mathbf{x}_{i}^{\prime}, t\right)$ is used in the previous section to image reflectors at and below $\partial \mathbb{D}_{i}$ from above. An image thus obtained is shown in Figure 15d, which represents the zero-offset reflectivity $R^{\cap}(\mathbf{x}, \mathbf{x}, 0)$ of the target zone, free of spurious reflections related to internal multiples in the overburden (amplitudes greater than $25 \%$ of the maximum amplitude have been clipped). Note the polarity change with respect to the image in Figure 14a.

The two imaging methods (from above and from below) use the same information, so the resulting images of $R^{\cup}(\mathbf{x}, \mathbf{x}, 0)$ and $R^{\cap}(\mathbf{x}, \mathbf{x}, 0)$ are not independent. Subtracting the two images (accounting for their opposite signs) will therefore not lead to a significant improvement of the $\mathrm{S} / \mathrm{N}$. Hence, for linear imaging applications, the two methods are equivalent (i.e., not complementary). However, for nonlinear applications, it can be useful to use the redatumed data $R^{\cup}\left(\mathbf{x}_{i}, \mathbf{x}_{i}^{\prime}, t\right)$ and $R^{\cap}\left(\mathbf{x}_{j}, \mathbf{x}_{j}^{\prime}, t\right)$ at two different depth levels $\partial \mathbb{D}_{i}$ and $\partial \mathbb{D}_{j}$ (with $x_{3, j}>x_{3, i}$ ). For example, these responses can be used as input for a new nonlinear imaging method, as introduced by Fleury and Vasconcelos (2012), Ravasi and Curtis (2013), and Vasconcelos (2013) or for full-waveform inversion (Virieux and Operto, 2009), to resolve the parameters of the target zone between these depth levels.

Van der Neut et al. (2013) discuss an alternative method to combine imaging from above with imaging from below. The first step involves Marchenko redatuming to two depth levels $\partial \mathbb{D}_{i}$ and $\partial \mathbb{D}_{j}$, yielding the Green's functions $G^{ \pm}\left(\mathbf{x}_{i}^{\prime}, \mathbf{x}_{0}^{\prime \prime}, t\right)$ and $G^{ \pm}\left(\mathbf{x}_{j}^{\prime}, \mathbf{x}_{0}^{\prime \prime}, t\right)$. These Green's functions are then used to simultaneously resolve the reflection responses to downgoing and upgoing waves of the target zone between $\partial \mathbb{D}_{i}$ and $\partial \mathbb{D}_{j}$. Note that because $G^{ \pm}\left(\mathbf{x}_{j}^{\prime}, \mathbf{x}_{0}^{\prime \prime}, t\right)$ contains reflections from objects below the target zone, this method uses essentially different information to image the target zone from below.

\section{COMPARISON WITH SOURCE-RECEIVER INTERFEROMETRY}

Because Marchenko imaging involves redatuming of sources as well as receivers, it has an interesting relation with source-receiver interferometry, as proposed by Curtis and Halliday (2010) and Halliday and Curtis (2010). We analyze this relation for the Marchenko scheme for imaging from below. We start this analysis by replacing $f_{2}^{+}$and $f_{2}^{-}$in equation 40 by their initial estimates $f_{2,0}^{+}$and $f_{2,0}^{-}$, respectively. According to equations $9,10,18,32$, and 33, we have

$$
f_{2,0}^{+}\left(\mathbf{x}_{j}, \mathbf{x}_{0}^{\prime \prime}, t\right)=-\int_{\partial \mathbb{D}_{0}} R^{\cup}\left(\mathbf{x}_{0}^{\prime \prime}, \mathbf{x}_{0},-t\right) * G_{\mathrm{d}}\left(\mathbf{x}_{0}, \mathbf{x}_{j}, t\right) \mathrm{d} \mathbf{x}_{0}
$$


and

$$
f_{2,0}^{-}\left(\mathbf{x}_{j}^{\prime}, \mathbf{x}_{0}^{\prime \prime}, t\right)=G_{\mathrm{d}}\left(\mathbf{x}_{0}^{\prime \prime}, \mathbf{x}_{j}^{\prime},-t\right),
$$

respectively. Here, the asterisk * denotes temporal convolution; for the sake of simplicity, we ignored the integration limit in the

a)
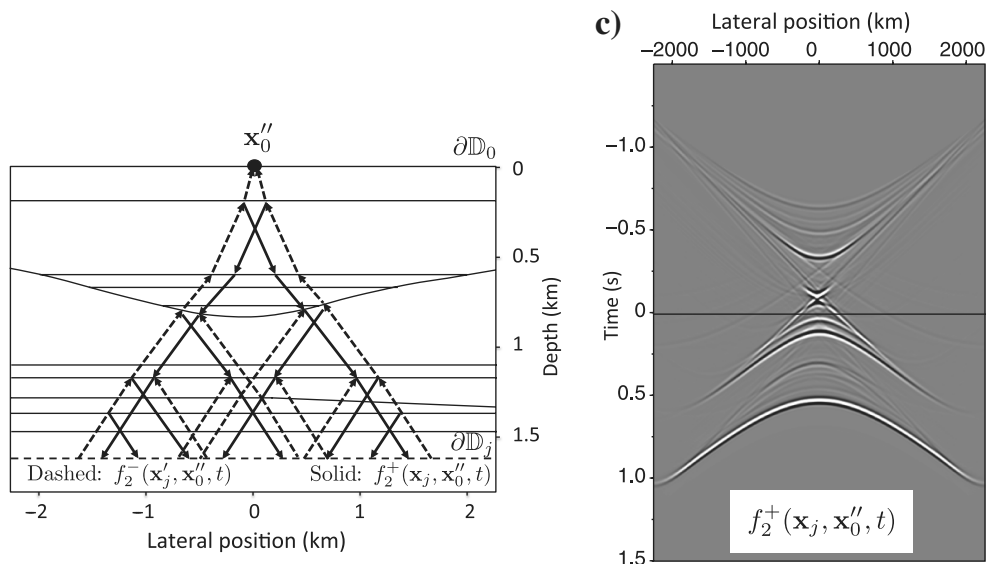

b) Lateral position $(\mathrm{km})$

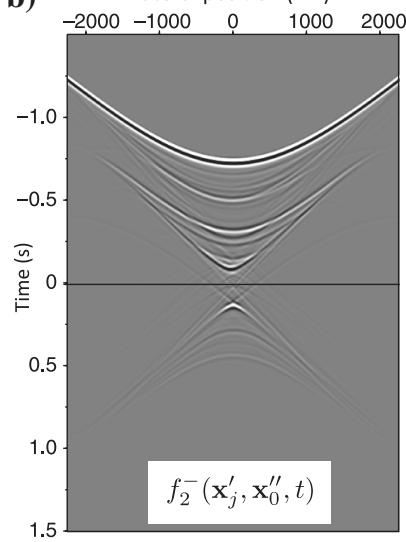

d)

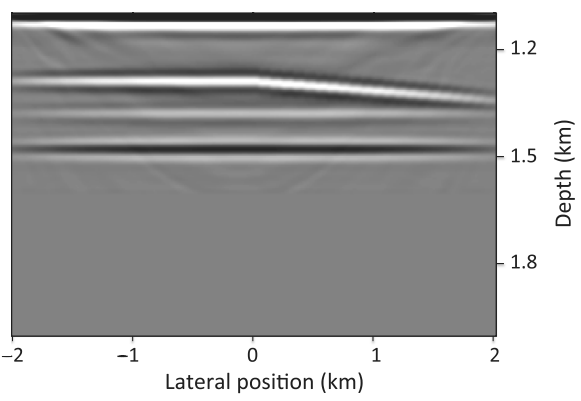

Figure 15. (a) Ray diagram of the focusing functions $f_{2}^{-}\left(\mathbf{x}_{j}^{\prime}, \mathbf{x}_{0}^{\prime \prime}, t\right)$ and $f_{2}^{+}\left(\mathbf{x}_{j}, \mathbf{x}_{0}^{\prime \prime}, t\right)$. (b) The retrieved focusing function $f_{2}^{-}\left(\mathbf{x}_{j}^{\prime}, \mathbf{x}_{0}^{\prime \prime}, t\right)$, for fixed $\mathbf{x}_{0}^{\prime \prime}$ and variable $\mathbf{x}_{j}^{\prime}$. (c) The retrieved focusing function $f_{2}^{+}\left(\mathbf{x}_{i}, \mathbf{x}_{0}^{\prime \prime}, t\right)$, for fixed $\mathbf{x}_{0}^{\prime \prime}$ and variable $\mathbf{x}_{j}$. (d) Marchenko image of the target area above $\partial \mathbb{D}_{j}$, obtained by resolving $R^{\cap}\left(\mathbf{x}_{j}, \mathbf{x}_{j}^{\prime}, t\right)$ from the focusing functions, followed by standard imaging.

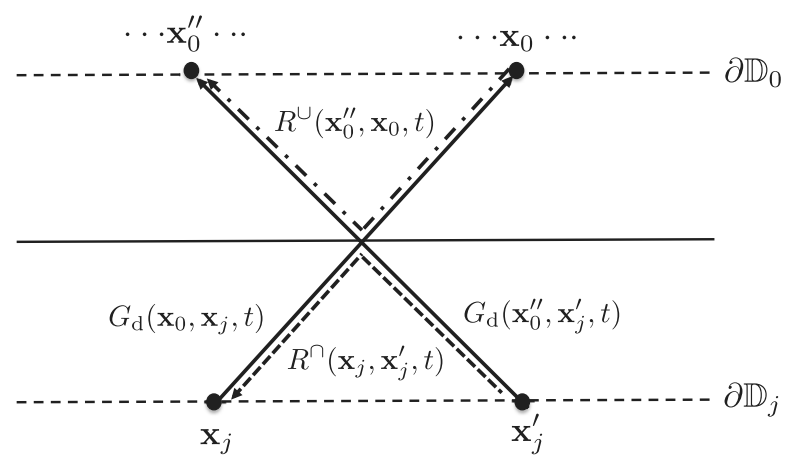

Figure 16. Source-receiver interferometry, for retrieving the primary reflection response from below (after Poliannikov et al., 2012). convolutional integral in equation 32. Resolving $R^{\cap}\left(\mathbf{x}_{j}, \mathbf{x}_{j}^{\prime}, t\right)$ from equation 40 involves MDD of $f_{2}^{+}$and $f_{2}^{-}$. If we approximate this MDD by crosscorrelation of the initial estimates $f_{2,0}^{+}$and $f_{2,0}^{-}$, we obtain

$$
\begin{aligned}
& R^{\cap}\left(\mathbf{x}_{j}, \mathbf{x}_{j}^{\prime}, t\right) \approx C\left(\mathbf{x}_{j}, \mathbf{x}_{j}^{\prime}, t\right) \\
& =\int_{\partial \mathbb{D}_{0}} f_{2,0}^{+}\left(\mathbf{x}_{j}, \mathbf{x}_{0}^{\prime \prime}, t\right) \\
& \quad * f_{2,0}^{-}\left(\mathbf{x}_{j}^{\prime}, \mathbf{x}_{0}^{\prime \prime},-t\right) \mathrm{d} \mathbf{x}_{0}^{\prime \prime} \\
& =-\int_{\partial \mathbb{D}_{0}} \int_{\partial \mathbb{D}_{0}} G_{\mathrm{d}}\left(\mathbf{x}_{0}^{\prime \prime}, \mathbf{x}_{j}^{\prime}, t\right) \\
& \quad * R^{\cup}\left(\mathbf{x}_{0}^{\prime \prime}, \mathbf{x}_{0},-t\right) \\
& * G_{\mathrm{d}}\left(\mathbf{x}_{0}, \mathbf{x}_{j}, t\right) \mathrm{d} \mathbf{x}_{0} \mathrm{~d} \mathbf{x}_{0}^{\prime \prime} .
\end{aligned}
$$

The double integral on the right side is a form of source-receiver interferometry. In particular, it represents the retrieval of the reflection response from below, as proposed by Poliannikov (2011) and Poliannikov et al. (2012). In their approach, the Green's functions represent responses of real sources in the subsurface. Figure 16 shows the configuration for the case of a single reflector. The indicated rays are the ones for which the traveltime of the integrand is stationary. According to equation 43, the two Green's functions and the time-reversed reflection response are convolved with each other; hence, the traveltimes along the solid rays in Figure 16 are added to each other and the traveltime along the dashed-dotted ray is subtracted from it. The resulting traveltime is that of the reflection response from below, indicated by the dashed ray.

This method works well for primary reflections, as is shown with numerical examples by Poliannikov (2011) and Poliannikov et al. (2012). However, equation 43 breaks down for multiple reflections. In contrast, the reflection response from below, obtained by inverting equation 40 with $f_{2}^{+}$and $f_{2}^{-}$obtained with the iterative Marchenko scheme, holds for primary as well as multiple reflections, as has been demonstrated with the numerical example in Figure 15.

\section{CONCLUSIONS}

We have shown that a decomposed version of the 3D Marchenko equation leads to an iterative scheme that enables the retrieval of the decomposed Green's functions $G^{-}\left(\mathbf{x}_{i}^{\prime}, \mathbf{x}_{0}^{\prime \prime}, t\right)$ and $G^{+}\left(\mathbf{x}_{i}^{\prime}, \mathbf{x}_{0}^{\prime \prime}, t\right)$ from reflection measurements at the acquisition surface $\partial \mathbb{D}_{0}$. Here, $\mathbf{x}_{0}^{\prime \prime}$ denotes the position of the Green's source at the surface, whereas $\mathbf{x}_{i}^{\prime}$ represents an arbitrary virtual-receiver position in the subsurface. Hence, the decomposed iterative Marchenko scheme accomplishes receiver redatuming. Subsequently, source redatuming is achieved by deconvolving (in a multidimensional sense) 
$G^{-}\left(\mathbf{x}_{i}, \mathbf{x}_{0}^{\prime \prime}, t\right)$ with $G^{+}\left(\mathbf{x}_{i}^{\prime}, \mathbf{x}_{0}^{\prime \prime}, t\right)$. This results in the redatumed response $R^{\cup}\left(\mathbf{x}_{i}, \mathbf{x}_{i}^{\prime}, t\right)$, with virtual sources $\left(\mathbf{x}_{i}^{\prime}\right)$ and virtual receivers $\left(\mathbf{x}_{i}\right)$ at depth level $\partial \mathbb{D}_{i}$ in the subsurface. This two-step redatuming procedure requires the same input as standard redatuming, namely the reflection response at the surface and an estimate of the direct arrivals between the surface and the redatuming level. However, whereas standard redatuming ignores the effects of internal multiples in the overburden, the redatuming procedure based on the Marchenko scheme and MDD properly accounts for internal multiples.

The redatumed response can be used in various ways for seismic imaging. We propose that these methods go together under the name "Marchenko imaging." In its most general form, angle-dependent reflectivity information is retrieved at any image position. More simple approaches lead to images of the zero-offset reflectivity, $R^{\cup}(\mathbf{x}, \mathbf{x}, 0)$, either by repeating the redatuming procedure for many depth levels $\partial \mathbb{D}_{i}$ and selecting the zero-offset, zero-time component, or by applying standard imaging to the redatumed response in a target zone below a fixed depth level $\partial \mathbb{D}_{i}$. In all cases, internal multiples contribute to the imaging process and the obtained image is free of spurious events related to these internal multiples. The nonrecursive character of Marchenko imaging implies that accumulation of errors is avoided. An important feature is that spurious reflections in a target zone are suppressed (as illustrated in Figure 14), without the need to resolve first the reflection properties of the overburden.

Marchenko imaging also has limitations. The conditions for the validity of the direct-wave assumption need further investigation. Moreover, the medium is assumed to be lossless. For media with small losses, it will probably suffice to apply loss corrections to the data prior to Marchenko imaging. However, for media with significant dissipation, more research is required. Losses due to mode conversion are also not covered by the current method. As in standard imaging, evanescent waves are excluded, which limits the spatial resolution. Sampling issues, for 2D and 3D data, also need further investigation. In its current form, our method requires that the reflection response is well sampled at the surface. Particularly for 3D data, this assumption is never fulfilled; hence, our method needs to be modified before it can be applied to realistic 3D data.

So far, we have only considered scalar waves. Representations 11 and 12 can straightforwardly be generalized for vectorial waves, but the derivation of the Marchenko equation for vectorial waves needs further investigation (particularly for elastodynamic waves, with different propagation velocities for $\mathrm{P}$ - and $\mathrm{S}$-waves, for which the causality arguments become more complex).

Despite the open questions, we believe that this work opens a new view on how to use information in internal multiple reflections to improve seismic imaging and monitoring of the earth's subsurface at different scales.

\section{ACKNOWLEDGMENTS}

This work is supported by the Netherlands Research Centre for Integrated Solid Earth Science. We greatly appreciate the constructive comments and suggestions of D.-J. van Manen, associate editor B. Biondi, and two anonymous reviewers, which helped improve this paper.

\section{APPENDIX A}

\section{DERIVATION OF RELATIONS BETWEEN GREEN'S FUNCTIONS, FOCUSING FUNCTIONS, AND REFLECTION AND TRANSMISSION RESPONSES}

We derive several relations between Green's functions, focusing functions, and reflection and transmission responses, using reciprocity theorems in the space-frequency domain.

We define the temporal Fourier transform of a space- and timedependent function $p(\mathbf{x}, t)$ as

$$
p(\mathbf{x}, \omega)=\int_{-\infty}^{\infty} p(\mathbf{x}, t) \exp (-j \omega t) \mathrm{d} t
$$

where $\omega$ is the angular frequency and $j$ the imaginary unit $(j=\sqrt{-1})$. To keep the notation simple, the same symbol is used for time- and frequency-domain functions (here $p$ ). In the space-frequency domain, we will make use of two reciprocity theorems for one-way wavefields at depth levels $\partial \mathbb{D}_{0}$ and $\partial \mathbb{D}_{i}$, enclosing a lossless medium (see Figures 1 and 2). Following a similar derivation as in Appendix B of Wapenaar and Berkhout (1989), we find the following reciprocity theorems for pressure-normalized one-way wavefields:

$$
\begin{aligned}
& -\int_{\partial \mathbb{D}_{0}} \rho^{-1}\left\{p_{A}^{+}\left(\partial_{3} p_{B}^{-}\right)+p_{A}^{-}\left(\partial_{3} p_{B}^{+}\right)\right\} \mathrm{d} \mathbf{x}_{0} \\
& =\int_{\partial \mathbb{D}_{i}} \rho^{-1}\left\{\left(\partial_{3} p_{A}^{+}\right) p_{B}^{-}+\left(\partial_{3} p_{A}^{-}\right) p_{B}^{+}\right\} \mathrm{d} \mathbf{x}_{i}
\end{aligned}
$$

and

$$
\begin{gathered}
-\int_{\partial \mathbb{D}_{0}} \rho^{-1}\left\{\left(p_{A}^{+}\right)^{*}\left(\partial_{3} p_{B}^{+}\right)+\left(p_{A}^{-}\right)^{*}\left(\partial_{3} p_{B}^{-}\right)\right\} \mathrm{d} \mathbf{x}_{0} \\
=\int_{\partial \mathbb{D}_{i}} \rho^{-1}\left\{\left(\partial_{3} p_{A}^{+}\right)^{*} p_{B}^{+}+\left(\partial_{3} p_{A}^{-}\right)^{*} p_{B}^{-}\right\} \mathrm{d} \mathbf{x}_{i},
\end{gathered}
$$

respectively. The asterisk denotes complex conjugation. The subscripts $A$ and $B$ refer to two independent acoustic states. The underlying assumptions are that the domain $\mathbb{D}$ between $\partial \mathbb{D}_{0}$ and $\partial \mathbb{D}_{i}$ is source-free, and the medium parameters in this domain are the same in both states. Furthermore, in equation A-3, the evanescent wavefields at $\partial \mathbb{D}_{0}$ and $\partial \mathbb{D}_{i}$ are neglected.

In the frequency domain, equation 3 reads

$$
\left.\partial_{3} G^{+}\left(\mathbf{x}, \mathbf{x}_{0}^{\prime \prime}, \omega\right)\right|_{x_{3}=x_{3,0}}=-\frac{1}{2} j \omega \rho\left(\mathbf{x}_{0}^{\prime \prime}\right) \delta\left(\mathbf{x}_{\mathrm{H}}^{\prime \prime}\right) \text {. }
$$

To derive equation 4 , substitute $p_{B}^{ \pm}(\mathbf{x}, \omega)=G^{ \pm}\left(\mathbf{x}, \mathbf{x}_{0}^{\prime \prime}, \omega\right)$ into equation A-2. Moreover, replace $\partial \mathbb{D}_{i}$ by $\partial \mathbb{D}_{m}$, where $\partial \mathbb{D}_{m}$ is a boundary that lies below all inhomogeneities, so that $p_{A}^{-}\left(\mathbf{x}_{m}, \omega\right)=$ $p_{B}^{-}\left(\mathbf{x}_{m}, \omega\right)=0$. This gives

$$
p_{A}^{-}\left(\mathbf{x}_{0}^{\prime \prime}, \omega\right)=\int_{\partial \mathbb{D}_{0}} R^{\cup}\left(\mathbf{x}_{0}^{\prime \prime}, \mathbf{x}_{0}, \omega\right) p_{A}^{+}\left(\mathbf{x}_{0}, \omega\right) \mathrm{d} \mathbf{x}_{0},
$$

with 


$$
R^{\cup}\left(\mathbf{x}_{0}^{\prime \prime}, \mathbf{x}_{0}, \omega\right)=\frac{\left.\partial_{3} G^{-}\left(\mathbf{x}, \mathbf{x}_{0}^{\prime \prime}, \omega\right)\right|_{x_{3}=x_{3,0}}}{\frac{1}{2} j \omega \rho\left(\mathbf{x}_{0}\right)}
$$

Transforming this back to the time domain gives equation 4 .

In the frequency domain, the focusing conditions 6 and 8 read

$$
\left.\partial_{3} f_{1}^{+}\left(\mathbf{x}, \mathbf{x}_{i}^{\prime}, \omega\right)\right|_{x_{3}=x_{3, i}}=-\frac{1}{2} j \omega \rho\left(\mathbf{x}_{i}^{\prime}\right) \delta\left(\mathbf{x}_{\mathrm{H}}-\mathbf{x}_{\mathrm{H}}^{\prime}\right)
$$

and

$$
\left.\partial_{3} f_{2}^{-}\left(\mathbf{x}, \mathbf{x}_{0}^{\prime \prime}, \omega\right)\right|_{x_{3}=x_{3,0}}=\frac{1}{2} j \omega \rho\left(\mathbf{x}_{0}^{\prime \prime}\right) \delta\left(\mathbf{x}_{\mathrm{H}}-\mathbf{x}_{\mathrm{H}}^{\prime \prime}\right),
$$

respectively. Substituting $p_{A}^{ \pm}(\mathbf{x}, \omega)=f_{1}^{ \pm}\left(\mathbf{x}, \mathbf{x}_{i}^{\prime}, \omega\right), \quad p_{B}^{ \pm}(\mathbf{x}, \omega)=$ $f_{2}^{ \pm}\left(\mathbf{x}, \mathbf{x}_{0}^{\prime \prime}, \omega\right)$ and $\left.\partial_{3} p_{A}^{-}(\mathbf{x}, \omega)\right|_{x_{3}=x_{3, i}}=\left.\partial_{3} p_{B}^{+}(\mathbf{x}, \omega)\right|_{x_{3}=x_{3,0}}=0$ into equations A-2 and A-3, using equations A-7 and A-8, gives

$$
f_{1}^{+}\left(\mathbf{x}_{0}^{\prime \prime}, \mathbf{x}_{i}^{\prime}, \omega\right)=f_{2}^{-}\left(\mathbf{x}_{i}^{\prime}, \mathbf{x}_{0}^{\prime \prime}, \omega\right)
$$

and

$$
-\left\{f_{1}^{-}\left(\mathbf{x}_{0}^{\prime \prime}, \mathbf{x}_{i}^{\prime}, \omega\right)\right\}^{*}=f_{2}^{+}\left(\mathbf{x}_{i}^{\prime}, \mathbf{x}_{0}^{\prime \prime}, \omega\right),
$$

respectively. Transforming these expressions back to the time domain yields equations 9 and 10, respectively.

To derive equations 11 and 12 consider the configurations in Figures 1 and 2. The Green's functions (state $B$ ) are defined in the actual medium (Figure 1), whereas the focusing functions (state $A)$ are defined in a reference configuration (Figure 2). Between depth levels $\partial \mathbb{D}_{0}$ and $\partial \mathbb{D}_{i}$, the medium is source-free and the medium parameters are the same in both states; hence, the conditions for reciprocity theorems A-2 and A-3 are satisfied. Substituting $p_{A}^{ \pm}(\mathbf{x}, \omega)=f_{1}^{ \pm}\left(\mathbf{x}, \mathbf{x}_{i}^{\prime}, \omega\right), p_{B}^{ \pm}(\mathbf{x}, \omega)=G^{ \pm}\left(\mathbf{x}, \mathbf{x}_{0}^{\prime \prime}, \omega\right)$, and $\left.\partial_{3} p_{A}^{-}(\mathbf{x}, \omega)\right|_{x_{3}=x_{3, i}}=0$ into equations $\mathrm{A}-2$ and A-3, using equations A-4, A-6, A-7, and A-8, yields

$$
\begin{aligned}
& \int_{\partial \mathbb{D}_{0}} R^{\cup}\left(\mathbf{x}_{0}^{\prime \prime}, \mathbf{x}_{0}, \omega\right) f_{1}^{+}\left(\mathbf{x}_{0}, \mathbf{x}_{i}^{\prime}, \omega\right) \mathrm{d} \mathbf{x}_{0}-f_{1}^{-}\left(\mathbf{x}_{0}^{\prime \prime}, \mathbf{x}_{i}^{\prime}, \omega\right) \\
& \quad=G^{-}\left(\mathbf{x}_{i}^{\prime}, \mathbf{x}_{0}^{\prime \prime}, \omega\right)
\end{aligned}
$$

and

$$
\begin{aligned}
& -\int_{\partial \mathbb{D}_{0}} R^{\cup}\left(\mathbf{x}_{0}^{\prime \prime}, \mathbf{x}_{0}, \omega\right)\left\{f_{1}^{-}\left(\mathbf{x}_{0}, \mathbf{x}_{i}^{\prime}, \omega\right)\right\}^{*} \mathrm{~d} \mathbf{x}_{0}+\left\{f_{1}^{+}\left(\mathbf{x}_{0}^{\prime \prime}, \mathbf{x}_{i}^{\prime}, \omega\right)\right\}^{*} \\
& \quad=G^{+}\left(\mathbf{x}_{i}^{\prime}, \mathbf{x}_{0}^{\prime \prime}, \omega\right),
\end{aligned}
$$

respectively. The inverse Fourier transforms of these expressions yield equations 11 and 12, respectively.

For the derivation of equation 15 consider Figure A-1. Here, $T\left(\mathbf{x}_{i}, \mathbf{x}_{0}^{\prime \prime}, t\right)$ represents the transmission response of the reference configuration, with $\mathbf{x}_{0}^{\prime \prime}$ just above $\partial \mathbb{D}_{0}$. Analogous to equation A-6, the Fourier transform of the transmission response is related to the Green's function of the reference configuration, according to

$$
T\left(\mathbf{x}_{i}, \mathbf{x}_{0}^{\prime \prime}, \omega\right)=\frac{\left.\partial_{3} \bar{G}^{+}\left(\mathbf{x}_{i}, \mathbf{x}^{\prime \prime}, \omega\right)\right|_{x_{3}^{\prime \prime}=x_{3,0}}}{\frac{1}{2} j \omega \rho\left(\mathbf{x}_{0}^{\prime \prime}\right)}
$$

Substituting $p_{A}^{+}\left(\mathbf{x}_{0}, \omega\right)=\delta\left(\mathbf{x}_{H}-\mathbf{x}_{\mathrm{H}}^{\prime \prime}\right), \quad p_{A}^{+}\left(\mathbf{x}_{i}, \omega\right)=T\left(\mathbf{x}_{i}, \mathbf{x}_{0}^{\prime \prime}, \omega\right)$, $p_{A}^{-}\left(\mathbf{x}_{i}, \omega\right)=0, p_{B}^{ \pm}(\mathbf{x}, \omega)=f_{2}^{ \pm}\left(\mathbf{x}, \mathbf{x}_{0}^{\prime}, \omega\right)$, and $\left.\partial_{3} p_{B}^{+}(\mathbf{x}, \omega)\right|_{x_{3}=x_{3,0}}=0$ into equation $\mathrm{A}-2$, using equation $\mathrm{A}-8$, gives

$$
\delta\left(\mathbf{x}_{\mathrm{H}}^{\prime}-\mathbf{x}_{\mathrm{H}}^{\prime \prime}\right)=\int_{\partial \mathbb{D}_{i}} \mathcal{T}\left(\mathbf{x}_{i}, \mathbf{x}_{0}^{\prime \prime}, \omega\right) f_{2}^{-}\left(\mathbf{x}_{i}, \mathbf{x}_{0}^{\prime}, \omega\right) \mathrm{d} \mathbf{x}_{i},
$$

where the modified transmission response $\mathcal{T}\left(\mathbf{x}_{i}, \mathbf{x}_{0}^{\prime \prime}, \omega\right)$ is given by

$$
\mathcal{T}\left(\mathbf{x}_{i}, \mathbf{x}_{0}^{\prime \prime}, \omega\right)=\frac{\left.\partial_{3} T\left(\mathbf{x}, \mathbf{x}_{0}^{\prime \prime}, \omega\right)\right|_{x_{3}=x_{3, i}}}{-\frac{1}{2} j \omega \rho\left(\mathbf{x}_{i}\right)}
$$

Hence, $f_{2}^{-}\left(\mathbf{x}_{i}, \mathbf{x}_{0}^{\prime \prime}, \omega\right)$ is the inverse of the modified transmission response $\mathcal{T}\left(\mathbf{x}_{i}, \mathbf{x}_{0}^{\prime \prime}, \omega\right)$ in the sense of equation A-14. In the time domain, this is formulated by equation 15 .

For the derivation of equation 40, we consider the reference configuration of Figure 2b for both states $A$ and $B$ (but with subscripts $i$ replaced by $j$ and a prime added to $\mathbf{x}$ ). For state $A$, we choose a Green's function in the reference configuration, according to $p_{A}^{ \pm}\left(\mathbf{x}^{\prime}, \omega\right)=\bar{G}^{ \pm}\left(\mathbf{x}^{\prime}, \mathbf{x}_{j}, \omega\right)$. Assuming the source at $\mathbf{x}_{j}$ is situated just below $\partial \mathbb{D}_{j}$, we have, analogous to equation A-4,

$$
\left.\partial_{3}^{\prime} \bar{G}^{-}\left(\mathbf{x}^{\prime}, \mathbf{x}_{j}, \omega\right)\right|_{x_{3}^{\prime}=x_{3, j}}=\frac{1}{2} j \omega \rho\left(\mathbf{x}_{j}\right) \delta\left(\mathbf{x}_{\mathrm{H}}^{\prime}-\mathbf{x}_{\mathrm{H}}\right),
$$

where $\partial_{3}^{\prime}$ stands for $\partial / \partial x_{3}^{\prime}$. For state $B$, we choose the second focusing function, hence $p_{B}^{ \pm}\left(\mathbf{x}^{\prime}, \omega\right)=f_{2}^{ \pm}\left(\mathbf{x}^{\prime}, \mathbf{x}_{0}^{\prime \prime}, \omega\right)$. Making these substitutions into equation A-2 (with a prime added to $\mathbf{x}_{j}$ ), taking into account that there are no downgoing waves at $\partial \mathbb{D}_{0}$, we obtain

$$
f_{2}^{+}\left(\mathbf{x}_{j}, \mathbf{x}_{0}^{\prime \prime}, \omega\right)=\int_{\partial \mathbb{D}_{j}} R^{\cap}\left(\mathbf{x}_{j}, \mathbf{x}_{j}^{\prime}, \omega\right) f_{2}^{-}\left(\mathbf{x}_{j}^{\prime}, \mathbf{x}_{0}^{\prime \prime}, \omega\right) \mathrm{d} \mathbf{x}_{j}^{\prime},
$$

with

$$
R^{\cap}\left(\mathbf{x}_{j}, \mathbf{x}_{j}^{\prime}, \omega\right)=\frac{\left.\partial_{3}^{\prime} \bar{G}^{+}\left(\mathbf{x}^{\prime}, \mathbf{x}_{j}, \omega\right)\right|_{x_{3}^{\prime}=x_{3, j}}}{-\frac{1}{2} j \omega \rho\left(\mathbf{x}_{j}^{\prime}\right)}
$$

Transforming equation A-17 back to the time domain gives equation 40 .

Homogeneous half-space

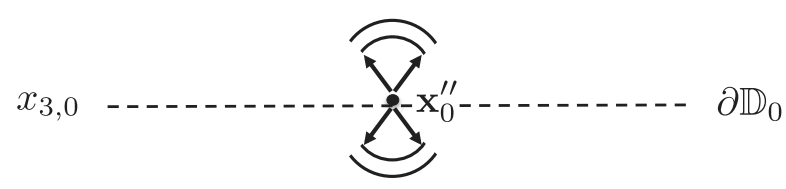

Actual inhomogeneous medium

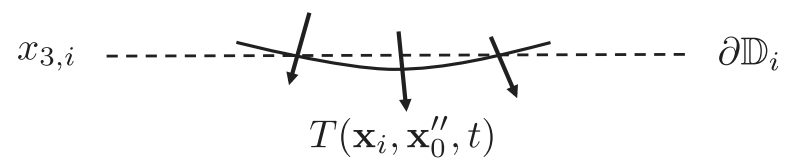

Reflection-free reference half-space

Figure A-1. Transmission response in the reference configuration. 


\section{APPENDIX B}

\section{RELATION BETWEEN THE DIFFERENT MARCHENKO EQUATIONS}

Marchenko equation 19 for the coda $M\left(\mathbf{x}_{i}^{\prime}, \mathbf{x}_{0}^{\prime \prime}, t\right)$ of the two-way focusing function $f_{2}\left(\mathbf{x}_{i}^{\prime}, \mathbf{x}_{0}^{\prime \prime}, t\right)$ is solved by the iterative scheme of equations 20 and 21 . The expression for the $k$ th iteration can alternatively be written as a series expansion, according to

$$
M_{k}\left(\mathbf{x}_{i}^{\prime}, \mathbf{x}_{0}^{\prime \prime}, t\right)=\sum_{l=0}^{k} N_{l}\left(\mathbf{x}_{i}^{\prime}, \mathbf{x}_{0}^{\prime \prime}, t\right),
$$

where

$$
\begin{aligned}
& N_{l}\left(\mathbf{x}_{i}^{\prime}, \mathbf{x}_{0}^{\prime \prime},-t\right)= \\
& \quad-\int_{\partial \mathbb{D}_{0}} \mathrm{~d} \mathbf{x}_{0} \int_{-t_{\mathrm{d}}^{e}\left(\mathbf{x}_{i}^{\prime}, \mathbf{x}_{0}\right)}^{t} R^{\cup}\left(\mathbf{x}_{0}^{\prime \prime}, \mathbf{x}_{0}, t-t^{\prime}\right) N_{l-1}\left(\mathbf{x}_{i}^{\prime}, \mathbf{x}_{0}, t^{\prime}\right) \mathrm{d} t^{\prime},
\end{aligned}
$$

for $t<t_{\mathrm{d}}\left(\mathbf{x}_{i}^{\prime}, \mathbf{x}_{0}^{\prime \prime}\right)$, whereas $N_{l}\left(\mathbf{x}_{i}^{\prime}, \mathbf{x}_{0}^{\prime \prime},-t\right)=0$ for $t \geq t_{\mathrm{d}}\left(\mathbf{x}_{i}^{\prime}, \mathbf{x}_{0}^{\prime \prime}\right)$. The expansion starts with

$$
N_{0}\left(\mathbf{x}_{i}^{\prime}, \mathbf{x}_{0}^{\prime \prime},-t\right)=M_{0}\left(\mathbf{x}_{i}^{\prime}, \mathbf{x}_{0}^{\prime \prime},-t\right) .
$$

The coupled system of Marchenko equations (equations 28 and 29) for $M^{+}\left(\mathbf{x}_{0}^{\prime \prime}, \mathbf{x}_{i}^{\prime}, t\right)$ (the coda of the one-way focusing function $\left.f_{1}^{+}\left(\mathbf{x}_{0}^{\prime \prime}, \mathbf{x}_{i}^{\prime}, t\right)\right)$ and $f_{1}^{-}\left(\mathbf{x}_{0}^{\prime \prime}, \mathbf{x}_{i}^{\prime}, t\right)$ is solved by the iterative scheme of equations 30-32. The expressions for the $m$ th iteration can also be rewritten as series expansions, according to

$$
M_{m}^{+}\left(\mathbf{x}_{0}^{\prime \prime}, \mathbf{x}_{i}^{\prime}, t\right)=\sum_{l=0}^{m} N_{2 l+1}\left(\mathbf{x}_{i}^{\prime}, \mathbf{x}_{0}^{\prime \prime}, t\right)
$$

and

$$
-f_{1, m}^{-}\left(\mathbf{x}_{0}^{\prime \prime}, \mathbf{x}_{i}^{\prime},-t\right)=\sum_{l=0}^{m} N_{2 l}\left(\mathbf{x}_{i}^{\prime}, \mathbf{x}_{0}^{\prime \prime}, t\right),
$$

respectively, and $M_{-1}^{+}\left(\mathbf{x}_{0}^{\prime \prime}, \mathbf{x}_{i}^{\prime}, t\right)=0$. Hence, $M_{m}^{+}\left(\mathbf{x}_{0}^{\prime \prime}, \mathbf{x}_{i}^{\prime}, t\right)$ and $-f_{1, m}^{-}\left(\mathbf{x}_{0}^{\prime \prime}, \mathbf{x}_{i}^{\prime},-t\right)$ can be obtained by selecting the odd and even terms, respectively, of expansion B-1 of the two-way coda $M_{k}\left(\mathbf{x}_{i}^{\prime}, \mathbf{x}_{0}^{\prime \prime}, t\right)$ (to be more specific, $M_{m}^{+}$consists of the odd terms of the expansion of $M_{k=2 m+1}$, whereas the time reversal of $-f_{1, m}^{-}$consists of the even terms of the expansion of $M_{k=2 m}$ ).

\section{REFERENCES}

Amundsen, L., 2001, Elimination of free-surface related multiples without need of the source wavelet: Geophysics, 66, 327-341, doi: 10.1190/1 .1444912 .

Bakulin, A., and R. Calvert, 2006, The virtual source method: Theory and case study: Geophysics, 71, no. 4, SI139-SI150, doi: 10.1190/1.2216190.

Behura, J., K. Wapenaar, and R. Snieder, 2012, Newton-Marchenko-Rose imaging: 82nd Annual International Meeting, SEG, Expanded Abstracts, doi: $10.1190 /$ segam2012-1531.1.

Berkhout, A. J., 1997, Pushing the limits of seismic imaging, Part I: Prestack migration in terms of double dynamic focusing: Geophysics, 62, 937953, doi: 10.1190/1.1444201.
Berkhout, A. J., and D. W. van Wulfften Palthe, 1979, Migration in terms of spatial deconvolution: Geophysical Prospecting, 27, 261-291, doi: 10 $.1111 / \mathrm{j} .1365-2478.1979 . t b 00970 . x$.

Berkhout, A. J., and D. J. Verschuur, 1997, Estimation of multiple scattering by iterative inversion. Part I: Theoretical considerations: Geophysics, 62, 1586-1595, doi: 10.1190/1.1444261.

Berkhout, A. J., and D. J. Verschuur, 2001, Seismic imaging beyond depth migration: Geophysics, 66, 1895-1912, doi: 10.1190/1.1487132.

Berkhout, A. J., and D. J. Verschuur, 2011, Full wavefield migration, utilizing surface and internal multiple scattering: 81st Annual International Meeting, SEG, Expanded Abstracts, 3212-3216.

Berryhill, J. R., 1979, Wave-equation datuming: Geophysics, 44, 13291344, doi: 10.1190/1.1441010.

Berryhill, J. R., 1984, Wave-equation datuming before stack: Geophysics, 49, 2064-2066, doi: 10.1190/1.1441620.

Broggini, F., and R. Snieder, 2012, Connection of scattering principles: A visual and mathematical tour: European Journal of Physics, 33, 593-613, doi: 10.1088/0143-0807/33/3/593.

Broggini, F., R. Snieder, and K. Wapenaar, 2014, Data-driven wave field focusing and imaging with multidimensional deconvolution: Numerical examples for reflection data with internal multiples: Geophysics, 79, no. 3, doi: 10.1190/geo2013-0307.1.

Brookes, D., 2011, Case studies in 3D interbed multiple elimination: The Leading Edge, 30, 914-918, doi: 10.1190/1.3626499.

Burridge, R., 1980, The Gelfand-Levitan, the Marchenko, and the GopinathSondhi integral equations of inverse scattering theory, regarded in the context of inverse impulse-response problems: Wave Motion, 2, 305-323, doi: 10.1016/0165-2125(80)90011-6.

Cao, W., S. M. Hanafy, G. T. Schuster, G. Zhan, and C. Boonyasiriwat, 2012, High-resolution and super stacking of time-reversal mirrors in locating seismic sources: Geophysical Prospecting, 60, 1-17, doi: 10.1111/j 1365-2478.2011.00957.x.

Chadan, K., and P. C. Sabatier, 1989, Inverse problems in quantum scattering theory: Springer.

Curtis, A., P. Gerstoft, H. Sato, R. Snieder, and K. Wapenaar, 2006, Seismic interferometry - Turning noise into signal: The Leading Edge, 25, 10821092, doi: 10.1190/1.2349814.

Curtis, A., and D. Halliday, 2010, Source-receiver wavefield interferometry: Physical Review E, 81, 046601, doi: 10.1103/PhysRevE.81.046601.

de Bruin, C. G. M., C. P. A. Wapenaar, and A. J. Berkhout, 1990, Angledependent reflectivity by means of prestack migration: Geophysics, $\mathbf{5 5}$, 1223-1234, doi: 10.1190/1.1442938.

de Hoop, A. T., 1995, Handbook of radiation and scattering of waves: Academic Press.

Fink, M., and F. Tanter, 2010, Multiwave imaging and super resolution: Physics Today, 63, no. 2, 28-33, doi: 10.1063/1.3326986.

Fleury, C., and I. Vasconcelos, 2012, Imaging condition for nonlinear scattering-based imaging: Estimate of power loss in scattering: Geophysics, 77, no. 1, S1-S18, doi: 10.1190/geo2011-0135.1.

Ge, D. B., 1987, An iterative technique in one-dimensional profile inversion: Inverse Problems, 3, 399-406, doi: 10.1088/0266-5611/3/3/009.

Halliday, D., and A. Curtis, 2010, An interferometric theory of sourcereceiver scattering and imaging: Geophysics, 75, no. 6, SA95-SA103, doi: $10.1190 / 1.3486453$.

Jakubowicz, H., 1998, Wave equation prediction and removal of interbed multiples: 68th Annual International Meeting, SEG, Expanded Abstracts, $1527-1530$

Kinneging, N. A., V. Budejicky, C. P. A. Wapenaar, and A. J. Berkhout, 1989, Efficient 2D and 3D shot record redatuming: Geophysical Prospecting, 37, 493-530, doi: 10.1111/j.1365-2478.1989.tb02220.x.

Lamb, G. L., 1980, Elements of soliton theory: John Wiley and Sons, Inc.

Lerosey, G., J. de Rosny, A. Tourin, and M. Fink, 2007, Focusing beyond the diffraction limit with far-field time reversal: Science, 315, 1120-1122, doi: $10.1126 /$ science. 1134824 .

Marchenko, V. A., 1955, Reconstruction of the potential energy from the phases of the scattered waves: Doklady Akademii Nauk SSSR, 104, 695-698 (in Russian).

Mehta, K., A. Bakulin, J. Sheiman, R. Calvert, and R. Snieder, 2007, Improving the virtual source method by wavefield separation: Geophysics, 72, no. 4, V79-V86, doi: 10.1190/1.2733020.

Poliannikov, O. V., 2011, Retrieving reflections by source-receiver wavefield interferometry: Geophysics, 76, no. 1, SA1-SA8, doi: 10.1190/1 3524241 .

Poliannikov, O. V., S. Rondenay, and L. Chen, 2012, Interferometric imaging of the underside of a subducting crust: Geophysical Journal International, 189, 681-690, doi: 10.1111/j.1365-246X.2012.05389.x.

Ravasi, M., and A. Curtis, 2013, Nonlinear scattering based imaging in elastic media: Theory, theorems, and imaging conditions: Geophysics, 78, no. 3, S137-S155, doi: 10.1190/geo2012-0286.1.

Sava, P. C., and S. Fomel, 2003, Angle-domain common-image gathers by wavefield continuation methods: Geophysics, 68, 1065-1074, doi: 10 .1190/1.1581078. 
Schuster, G. T., and M. Zhou, 2006, A theoretical overview of model-based and correlation-based redatuming methods: Geophysics, 71, no. 4, SI103SI1 10, doi: 10.1190/1.2208967.

Slob, E., K. Wapenaar, F. Broggini, and R. Snieder, 2014, Seismic reflector imaging using internal multiples with Marchenko-type equations: Geophysics, 79, no. 2, S63-S76, doi: 10.1190/geo2013-0095.1.

Snieder, R., K. Wapenaar, and K. Larner, 2006, Spurious multiples in seismic interferometry of primaries: Geophysics, 71, no. 4, SI111-SI124, doi: 10.1190/1.2211507.

Ten Kroode, F., 2002, Prediction of internal multiples: Wave Motion, 35, 315-338, doi: 10.1016/S0165-2125(01)00109-3.

van der Neut, J., E. Slob, K. Wapenaar, J. Thorbecke, R. Snieder, F. Broggini, J. Behura, and S. Singh, 2013, Interferometric redatuming of autofocused primaries and internal multiples: 83rd Annual International Meeting, SEG, Expanded Abstracts, 4589-4594.

van der Neut, J., J. Thorbecke, K. Mehta, E. Slob, and K. Wapenaar, 2011 Controlled-source interferometric redatuming by crosscorrelation and multidimensional deconvolution in elastic media: Geophysics, 76, no. 4, SA63-SA76, doi: 10.1190/1.3580633.

van Groenestijn, G. J. A., and D. J. Verschuur, 2009, Estimation of primaries and near-offset reconstruction by sparse inversion: Marine data applications: Geophysics, 74, no. 6, R119-R128, doi: 10.1190/1 .3213532 .

Vasconcelos, I., 2013, Source-receiver, reverse-time imaging of dual-source, vector-acoustic seismic data: Geophysics, 78, no. 2, WA123-WA145, doi: 10.1190/geo2012-0300.1.

Verschuur, D. J., A. J. Berkhout, and C. P. A. Wapenaar, 1992, Adaptive surface-related multiple elimination: Geophysics, 57, 1166-1177, doi: 10.1190/1.1443330.
Virieux, J., and S. Operto, 2009, An overview of full-waveform inversion in exploration geophysics: Geophysics, 74, no. 6, WCC1-WCC26, doi: 10 $.1190 / 1.3238367$.

Wapenaar, C. P. A., and A. J. Berkhout, 1989, Elastic wave field extrapolation: Elsevier.

Wapenaar, K., J. Fokkema, M. Dillen, and P. Scherpenhuijsen, 2000, Oneway acoustic reciprocity and its applications in multiple elimination and time-lapse seismics: 70th Annual International Meeting, SEG, Expanded Abstracts, 2377-2380.

Wapenaar, K., J. Thorbecke, J. van der Neut, F. Broggini, E. Slob, and R. Snieder, 2014, Green's function retrieval from reflection data, in absence of a receiver at the virtual source position: Journal of the Acoustical Society of America, 134, doi: 10.1121/1.4869083.

Wapenaar, K., J. Thorbecke, J. van der Neut, E. Slob, F. Broggini, J. Behura, and R. Snieder, 2012, Integrated migration and internal multiple elimination: 82nd Annual International Meeting, SEG, Expanded Abstracts SPMUL 1.5 .

Weglein, A. B., F. A. Gasparotto, P. M. Carvalho, and R. H. Stolt, 1997, An inverse-scattering series method for attenuating multiples in seismic reflection data: Geophysics, 62, 1975-1989, doi: 10.1190/1.1444298.

Weglein, A. B., S. Y. Hsu, P. Terenghi, X. Li, and R. H. Stolt, 2011, Multiple attenuation: Recent advances and the road ahead (2011): The Leading Edge, 30, 864-875, doi: 10.1190/1.3626494.

Williams, E. G., and J. D. Maynard, 1980, Holographic imaging without the wavelength resolution limit: Physical Review Letters, 45, 554-557, doi: 10.1103/PhysRevLett.45.554. 